ARTICLE OPEN

\title{
The PIWI protein Aubergine recruits eIF3 to activate translation in the germ plasm
}

Anne Ramat ${ }^{1}$, Maria-Rosa Garcia-Silva ${ }^{1}$, Camille Jahan ${ }^{1}$, Rima Naït-Saïdi ${ }^{1}$, Jérémy Dufourt (iD ${ }^{1,5}$, Céline Garret ${ }^{1}$, Aymeric Chartier ${ }^{1}$, Julie Cremaschi ${ }^{1}$, Vipul Patel ${ }^{1}$, Mathilde Decourcelle (iD) ${ }^{2}$, Amandine Bastide ${ }^{3}$, François Juge (iD) ${ }^{4}$ and Martine Simonelig (iD ${ }^{1}$

Piwi-interacting RNAs (piRNAs) and PIWI proteins are essential in germ cells to repress transposons and regulate mRNAs. In Drosophila, piRNAs bound to the PIWI protein Aubergine (Aub) are transferred maternally to the embryo and regulate maternal mRNA stability through two opposite roles. They target mRNAs by incomplete base pairing, leading to their destabilization in the soma and stabilization in the germ plasm. Here, we report a function of Aub in translation. Aub is required for translational activation of nanos mRNA, a key determinant of the germ plasm. Aub physically interacts with the poly(A)-binding protein (PABP) and the translation initiation factor elF3. Polysome gradient profiling reveals the role of Aub at the initiation step of translation. In the germ plasm, PABP and elF3d assemble in foci that surround Aub-containing germ granules, and Aub acts with elF3d to promote nanos translation. These results identify translational activation as a new mode of mRNA regulation by Aub, highlighting the versatility of PIWI proteins in mRNA regulation.

Cell Research (2020) 30:421-435; https://doi.org/10.1038/s41422-020-0294-9

\section{INTRODUCTION}

Translational control is a widespread mechanism to regulate gene expression in many biological contexts. This regulation has an essential role during early embryogenesis, before transcription of the zygotic genome has actually started. In Drosophila, embryonic patterning depends on the translational control of a small number of maternal mRNAs. ${ }^{1}$ Among them, nanos (nos) mRNA encodes a key posterior determinant required for abdominal segmentation and development of the germline. ${ }^{2}$ nos mRNA is present in the whole embryo, but a small proportion accumulates at the posterior pole in the germ plasm, a specialized cytoplasm in which the germline develops. $^{3,4}$ Localization and translational control of nos mRNA are linked, such that the pool of nos mRNA present in the bulk of the embryo is translationally repressed, whereas the pool of nos mRNA localized in the germ plasm is translationally activated to produce a Nos protein gradient from the posterior pole. ${ }^{3,5,6}$ Both repression of nos mRNA translation in the bulk of the embryo and activation in the germ plasm are required for embryonic development.

The coupling between mRNA localization and translational control depends in part on the implication of the same factors in both processes. The Smaug (Smg) RNA binding protein specifically recognizes nos mRNA through binding to two Smaug recognition elements (SRE) in its $3^{\prime} \mathrm{UTR}^{7,8} \mathrm{Smg}$ is both a translational repressor of nos, and a localization factor through its role in mRNA deadenylation and decay in the bulk of the embryo, by recruitment of the CCR4-NOT deadenylation complex. ${ }^{7-9}$ Smg directly interacts with the Oskar (Osk) protein that is specifically synthesized at the posterior pole of oocytes and embryos and drives germ plasm assembly., ${ }^{70}$ Smg interaction with Osk prevents Smg binding to nos mRNA, thus contributing to relieving both Smg-dependent translational repression and mRNA decay in the germ plasm. ${ }^{7,9,11}$ Osk is therefore a key player in the switch of nos and other germ cell mRNA regulation between soma and germ plasm of the embryo.

More recently, we have demonstrated the role of Aubergine (Aub) in the localization of germ cell mRNAs to the germ plasm. ${ }^{12,13}$ Aub is one of the three PIWI proteins in Drosophila. PIWI proteins belong to a specific clade of Argonaute proteins that bind 23-30 nucleotides (nt)-long small RNAs referred to as Piwiinteracting RNAs (piRNAs). ${ }^{14,15}$ piRNAs and PIWI proteins have an established role in the repression of transposable elements in the germline of animals. piRNAs target transposable element mRNAs through complementarity and guide interaction with PIWI proteins that, in turn, cleave targeted mRNAs through their endonucleolytic activity. In addition to this role, piRNAs have a conserved function in the regulation of cellular mRNAs in various biological contexts. ${ }^{16}$ In the Drosophila embryo, Aub loaded with piRNAs produced in the female germline is present both at low levels in the bulk of the embryo and at higher levels in the germ plasm. ${ }^{17,18}$ Aub binds maternal germ cell mRNAs through incomplete base pairing with piRNAs. ${ }^{12,18,19}$ Aub binding to these mRNAs induces their decay in the bulk of the embryo, either by direct cleavage or recruitment together with Smg of the CCR4NOT deadenylation complex. ${ }^{12,18}$ In contrast, in the germ plasm

\footnotetext{
${ }^{1}$ mRNA Regulation and Development, Institute of Human Genetics, UMR9002 CNRS-Univ Montpellier, 141 rue de la Cardonille, 34396 Montpellier Cedex 5, France; ${ }^{2}$ BioCampus Montpellier, CNRS, INSERM, Univ Montpellier, Montpellier, France; ${ }^{3}$ IGF, Univ Montpellier, CNRS, INSERM, Montpellier, France and ${ }^{4}$ Institut de Génétique Moléculaire de Montpellier, Univ Montpellier, CNRS, Montpellier, France

Correspondence: Martine Simonelig (Martine.Simonelig@igh.cnrs.fr)

${ }^{5}$ Present address: Institut de Génétique Moléculaire de Montpellier, Univ Montpellier, CNRS, Montpellier, France

These authors contributed equally: Anne Ramat, Maria-Rosa Garcia-Silva
}

Received: 29 November 2019 Accepted: 11 February 2020

Published online: 4 March 2020 

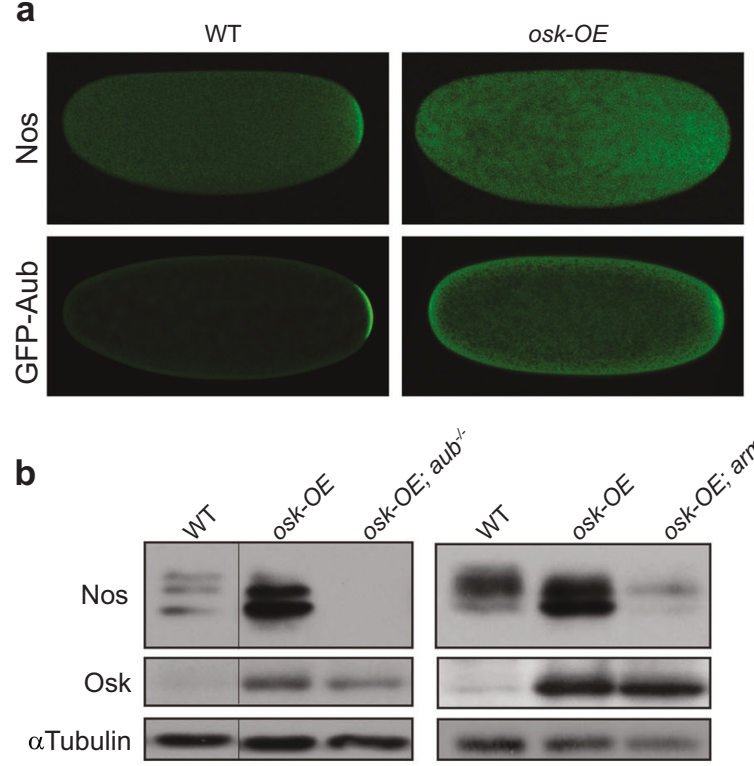

C
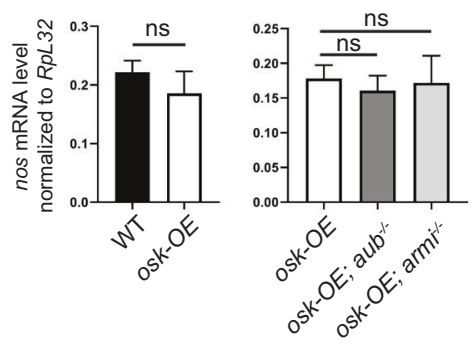
d Aub crosslink
sites
nos nos
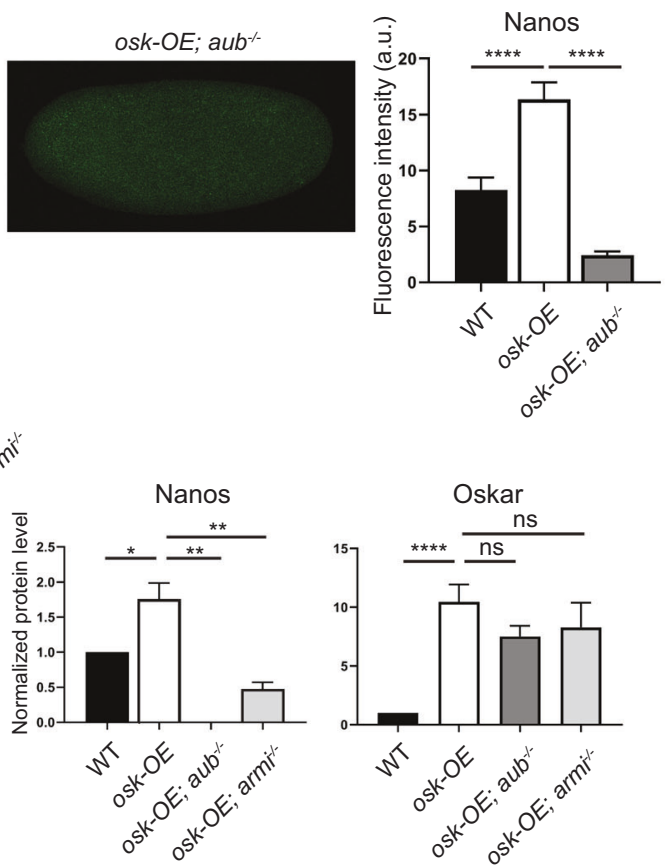

osk-OE : UASp-osk/+; nos-Gal4/+

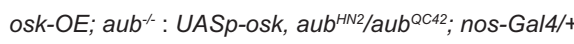

osk-OE; armi' : UASp-osk/+; armi ${ }^{72.1}$ nos-Gal4/armi ${ }^{1}$

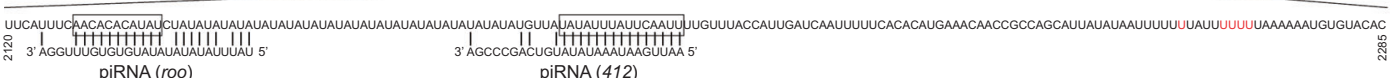
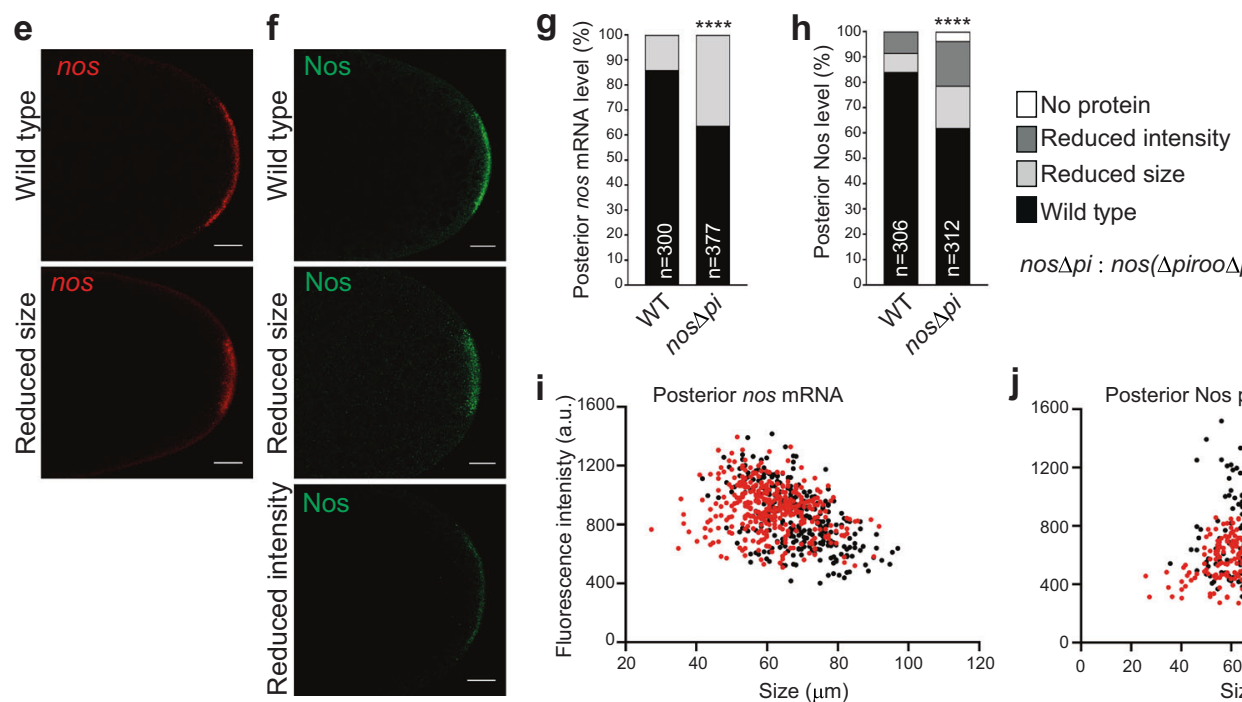

$\square$ Reduced size

Wild type

nos $\Delta p i: \operatorname{nos}\left(\Delta\right.$ piroospi412)/+; nos ${ }^{B N / B N x}$
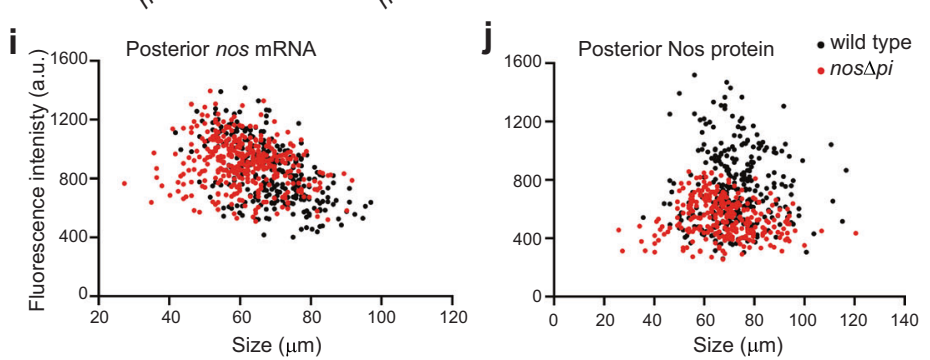

Aub recruits Wispy, the germline-specific cytoplasmic poly(A) polymerase, leading to poly $(A)$ tail elongation and stabilization of Aub-bound mRNAs. ${ }^{13}$ Thus, Aub and piRNAs play a central role in the localization of germ cell mRNAs through two opposite functions in mRNA stability: mRNA destabilization in the bulk of the embryo and stabilization in the germ plasm. The role of piRNAs and PIWI proteins in cellular mRNA regulation in other contexts, including mouse spermiogenesis and sex determination in Bombyx, also depends on their function in the regulation of mRNA stability. ${ }^{20-24}$ 
Fig. 1 Aub and Armi are required for nos mRNA translation. a Immunostaining of WT, osk-OE and osk-OE; aub ${ }^{-1-}$ embryos with anti-Nos antibody (top panels). The genotypes are indicated. Fluorescence quantification was performed using the ImageJ software with 5-6 embryos per genotype. Error bars represent SEM. ${ }^{* * *} P<0.0001$ using the unpaired Student's $t$-test. Immunostaining of UASp-GFP-Aub nos-Gal4 and UASp-osk/+; UASp-GFP-Aub nos-Gal4/+ embryos with anti-GFP antibody, showing the distribution of Aub protein (bottom panels). Posterior is to the right. b Western blots of WT, osk-OE, osk-OE; aub ${ }^{-/-}$and osk-OE; armi ${ }^{-1-}$ embryos revealed with anti-Nos, anti-Osk and anti- $\alpha-T u b u l i n$ antibodies. $\alpha$-Tubulin was used as a loading control. Quantification was performed using the ImageJ software with 3-6 biological replicates. Error bars represent SEM. ${ }^{* * *} P<0.0001,{ }^{*} P<0.01,{ }^{*} P<0.05$, ns not significant, using the unpaired Student's $t$-test. c Quantification of nos mRNA using RT-qPCR in WT, osk-OE, osk-OE; aub ${ }^{-1-}$ and osk-OE; armi ${ }^{-1-}$ embryos. mRNA levels were normalized with RpL32 mRNA. Quantification of 4-8 biological replicates. Error bars represent SEM. ns not significant, using the unpaired Student's $t$-test. d Schematic representation of nos mRNA and 3'UTR targeting with piRNAs. Thin boxes are 5'UTR and $3^{\prime}$ UTR, lines are introns and thick boxes are exons. Clusters of Aub crosslink sites are indicated in red. ${ }^{12}$ The sequence of the region with the strongest crosslink sites and base pairing with representative roo and 412 piRNAs are shown. The deletions overlapping with the piRNA target sites in the nos( $\triangle$ piroo $\Delta$ pi412) transgene are boxed. ${ }^{18}$ Aub crosslinked nt are in red. e, $\mathbf{f}$ nos smFISH (e) and immunostaining with anti-Nos antibody (f) of wild-type and nos $\left(\triangle\right.$ piroo $\triangle$ pi412)/+; nos ${ }^{B N / B N x}$ embryos. Posterior of embryos with the three types of staining: wild type, reduced size or reduced intensity, are shown. Scale bars, $20 \mu \mathrm{m}$. $\mathbf{g}$-j Quantification of posterior staining shown in e and $\mathbf{f}$ using the ImageJ software. For each genotype, the percentage of embryos with each staining category was recorded for nos mRNA (g) and Nos protein (h). ${ }^{* * * *} P<0.0001$ using the $\chi^{2}$ test. Scatter plots of size and fluorescence intensity of posterior staining for each embryo, for nos mRNA (i) and Nos protein (j). Two-way ANOVA showed significant difference $(P<0.001)$ in fluorescence intensity of posterior staining for nos mRNA and Nos protein between genotypes.

Here, we describe translational activation as a new mechanism of mRNA regulation by piRNAs and PIWI proteins. Using ectopic expression of Osk in the whole embryo to mimic the germ plasm, we show that Aub and piRNAs are required for nos mRNA translation. Mass spectrometry analysis of Aub interactors in early embryos identifies several components of the translation machinery, including translation initiation factors. We find that Aub physically interacts with the poly(A)-binding protein (PABP) and several subunits of the translation initiation complex elF3. Furthermore, PABP and elF3d accumulate in foci that assemble around and partially overlap with Aub-containing germ granules in the germ plasm. Polysome gradient analysis indicates that Aub activates translation at the initiation step. Finally, functional experiments involving the concomitant decrease of Aub and elF3d show that both proteins act together in nos mRNA translation in the germ plasm. These results identify translational activation as a new level of mRNA regulation by PIWI proteins. Moreover, they expand the role of the general elF3 translation initiation complex in translation regulatory mechanisms required for developmental processes.

\section{RESULTS}

Aub is required for nos mRNA translation

Only a low amount (4\%) of nos mRNA is localized to the germ plasm and actually translated. ${ }^{3,4}$ nos mRNA stabilization and translation in the germ plasm depend on the presence of Osk. Therefore, we ectopically expressed Osk in the whole embryo using UASp-osk ${ }^{25}$ and the germline-specific driver nos-Gal4, to increase translated nos mRNA levels and address the mechanisms of translational activation. Osk overexpression (osk-OE) in embryos from UASp-osk/+; nos-Gal4/+ females led to increased and ectopic Nos protein synthesis in whole embryo (Fig. 1a). Quantification of Nos protein levels in osk-OE embryos, either following Nos visualization using immunostaining or western blot, revealed a 2-fold increase compared to wild-type (WT) embryos (Fig. 1a, b). In contrast, nos mRNA levels quantified using RT-qPCR were similar in osk-OE and WT embryos (Fig. 1c). This is consistent with the presence of high amounts of nos mRNA in the bulk of embryos, and nos spatial regulation depending mostly on translational control at these stages (0-2 $\mathrm{h}$ embryos). Therefore, Osk overexpression in $0-2 \mathrm{~h}$ embryos led to ectopic translational activation of nos mRNA without changes in nos mRNA levels.

Aub protein is present at low levels in the bulk of WT embryos and highly accumulates in the germ plasm. ${ }^{18}$ Ectopic expression of Osk led to an homogeneous redistribution of Aub in the embryo (Fig. 1a). Strikingly, the lack of Aub in osk-OE embryos resulted in the lack of Nos protein synthesis (Fig. 1a, b), although nos mRNA levels were not decreased (Fig. 1c). This result suggested that Aub was required for nos mRNA translational activation in the presence of Osk. Importantly, the level of Osk protein was not significantly affected by aub mutation, indicating that the lack of Nos protein did not result from the lack of Osk (Fig. 1b). Of note, the UASp-osk transgene almost exclusively overexpressed the long Osk isoform of the two isoforms, Short-Osk and Long-Osk (Supplementary information, Fig. S1a). Long-Osk can induce germ plasm assembly when overexpressed although less actively than Short-Osk. ${ }^{26}$ Long-Osk levels were poorly affected by aub mutations, making this UASp-osk transgene a useful tool to address direct nos mRNA regulation by Aub and piRNAs (Fig. 1b; Supplementary information, Fig. S1a).

We analyzed the role of Armitage (Armi), another component of the piRNA pathway with a prominent role in piRNA biogenesis, ${ }^{27}$ in nos mRNA translational activation. Nos protein levels were strongly reduced in osk-OE; armi ${ }^{-1-}$ embryos, compared to osk-OE embryos, although again, the levels of Osk protein were not significantly decreased (Fig. 1b). nos mRNA levels quantified using RT-qPCR remained unaffected by armi mutation (Fig. 1c), revealing a role of Armi in nos mRNA translational control. Armi does not localize to the germ plasm. ${ }^{13}$ Instead the defect in nos mRNA translational activation in armi mutant might depend on highly reduced piRNA levels in this mutant, ${ }^{27}$ suggesting that piRNAs were required in Aub binding to nos mRNA for its role in translational activation. This is consistent with Aub iCLIP assays showing that an Aub double point mutant in the PAZ domain, Aub $^{A A}$ that is unable to load piRNAs, was also unable to bind mRNAs. ${ }^{12}$ To confirm the role of piRNAs in Aub-dependent translational activation of nos, we took advantage of the nos $(\triangle$ piroo $\Delta$ pi412) transgene, in which two piRNA target sites (from roo and 412 transposable elements) in close proximity to a prominent Aub-binding site in nos $3^{\prime}$ UTR have been deleted ${ }^{12,18}$ (Fig. 1d). We have shown before that deletion of these piRNA target sites affected nos mRNA localization to the germ plasm, without affecting its level. ${ }^{13}$ Using single molecule fluorescence in situ hybridization (smFISH), we confirmed the posterior localization defect of nos mRNA from this transgene: $36 \%$ of embryos showed a reduced domain of posterior localization (Fig. 1e, g, i). As previously reported, this defect was moderate because several Aub-binding sites remained unaffected in the nos (Apiroo $\Delta$ pi412) transgene (Fig. 1d). ${ }^{13}$ Recording nos mRNA translation in nos( $\triangle$ piroo $\triangle$ pi412)/+; nos ${ }^{B N / B N x}$ embryos using immunostaining showed a similar percentage of embryos (38\%) with reduced protein accumulation (Fig. 1f, h, j). However, protein synthesis appeared to be more affected than mRNA localization in these embryos since $3.8 \%$ of them did not produce any Nos protein, a defect (no localization) that did not occur with nos mRNA (Fig. 1e, g). In addition, when taking into account all embryos, immunofluorescence intensity was reduced in nos 

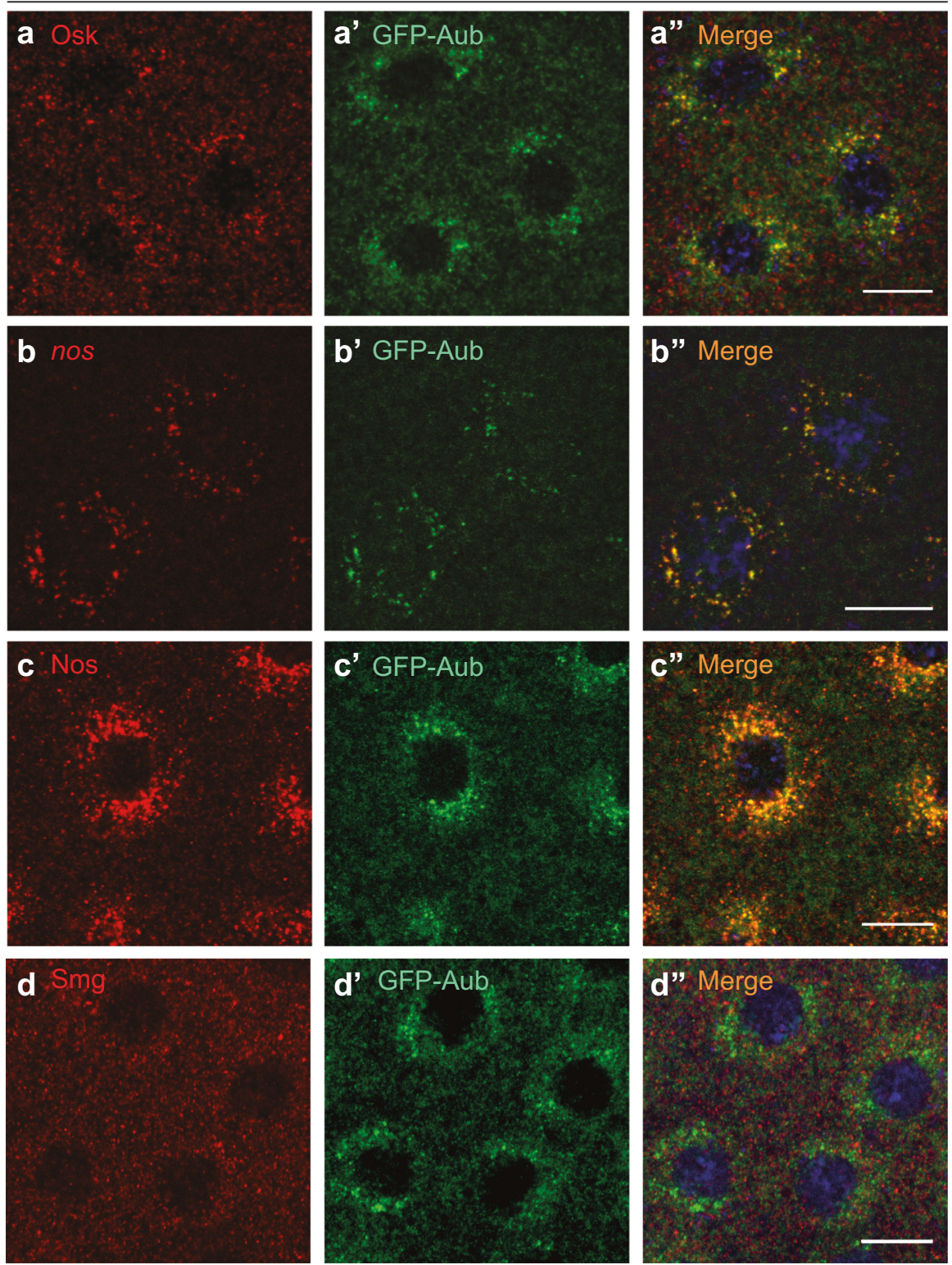

e

\begin{tabular}{|l|c|c|}
\cline { 2 - 3 } \multicolumn{1}{c|}{} & $\begin{array}{c}\% \text { Colocalization in } \\
\text { granules }(>0.5 \mu \mathrm{m})\end{array}$ & $\begin{array}{c}\% \text { Colocalization in } \\
\text { foci }(>0.2 \mu \mathrm{m})\end{array}$ \\
\hline Aub in Osk & $70 \% \pm 6(\mathrm{n}=66)$ & $3.3 \% \pm 0.8(\mathrm{n}=446)$ \\
\hline Osk in Aub & $77.2 \% \pm 7(\mathrm{n}=64)$ & $2.7 \% \pm 0.6(\mathrm{n}=493)$ \\
\hline Aub in $n o s$ & $77.2 \% \pm 1(\mathrm{n}=156)$ & N/A \\
\hline nos in Aub & $81.4 \% \pm 2(\mathrm{n}=147)$ & N/A \\
\hline Aub in Nos & $71.6 \% \pm 5(\mathrm{n}=102)$ & $9.8 \% \pm 5(\mathrm{n}=138)$ \\
\hline Nos in Aub & $78.4 \% \pm 8(\mathrm{n}=104)$ & $9.4 \% \pm 4(\mathrm{n}=128)$ \\
\hline Aub in Smg & $1.5 \% \pm 0.5(\mathrm{n}=609)$ & $0.8 \% \pm 0.1(\mathrm{n}=1068)$ \\
\hline Smg in Aub & $1.9 \% \pm 0.6(\mathrm{n}=512)$ & $0.9 \% \pm 0.2(\mathrm{n}=1017)$ \\
\hline
\end{tabular}

Fig. 2 Ectopic expression of Osk nucleates RNA granules related to germ granules in the soma. a-d" Immunostaining of UASp-osk/+; UASpGFP-Aub nos-Gal4/+ embryos with anti-Osk (red) and anti-GFP (green) to visualize Aub (a-a"); anti-Nos (red) and anti-GFP (green) (c-c"); and anti-Smg (red) and anti-GFP (green) (d-d"); and smFISH of embryos with the same genotype revealing nos mRNA and GFP-Aub through GFP fluorescence (b-b"). DNA was visualized using DAPI. Scale bars, $10 \mu \mathrm{m}$. e Quantification of colocalization of immunostaining and smFISH shown in a-d", using the Imaris software. Granules $(>0.5 \mu \mathrm{m})$ and foci $(>0.2 \mu \mathrm{m})$ were quantified around nuclei and in the cytoplasm between nuclei, respectively. 
$\left(\Delta\right.$ piroo $\Delta$ pi412)/+; $\operatorname{nos}^{B N / B N x}$ embryos compared to WT, again a defect that did not occur with smFISH (Fig. 1i, j). Therefore, deletion of piRNA target sites in nos mRNA affected Nos protein synthesis, in addition to reducing mRNA localization.

These data are consistent with a direct role of Aub and piRNAs in nos mRNA translation through piRNA-guided binding of Aub to nos. In this hypothesis, a piRNA pathway component specifically involved in transposable element regulation should not interfere with nos mRNA translational control. We used Panoramix (Panx), a key factor in Piwi-dependent transcriptional silencing of transposable elements, which acts downstream of Piwi and has no function in piRNA biogenesis. ${ }^{28,29}$ panx mutants had no effect on
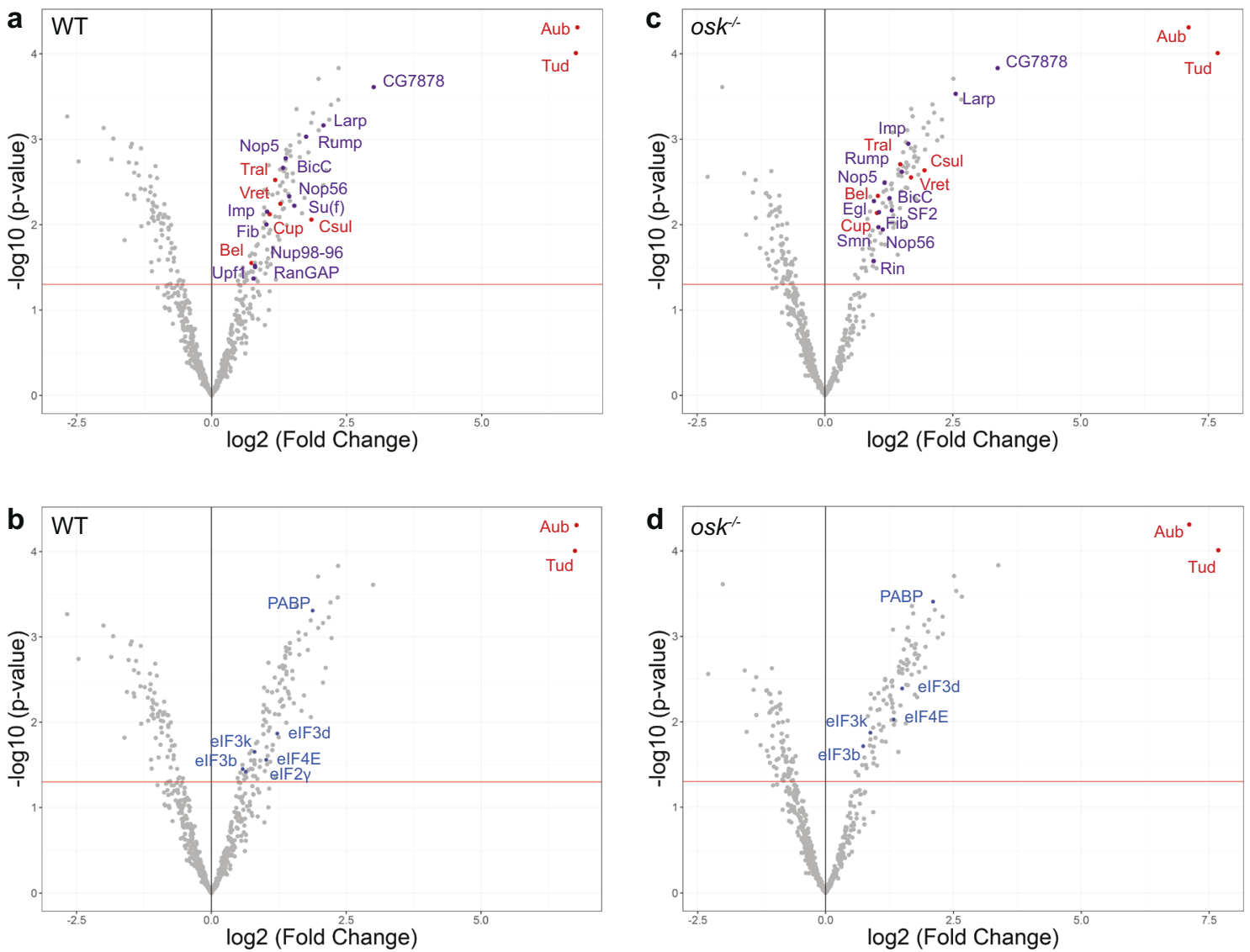

Aub interactors

\begin{tabular}{lcc}
\hline GO terms (104 genes) & p-value & Matches \\
\hline Biological process & & \\
\hline Cytoplasmic translation & $5.168318 \mathrm{e}-68$ & 51 \\
Translation & $1.644336 \mathrm{e}-35$ & 60 \\
Peptide Biosynthesis process & $2.109374 \mathrm{e}-34$ & 60
\end{tabular}

f

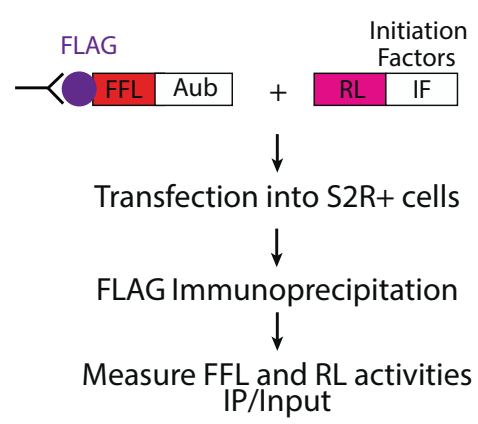

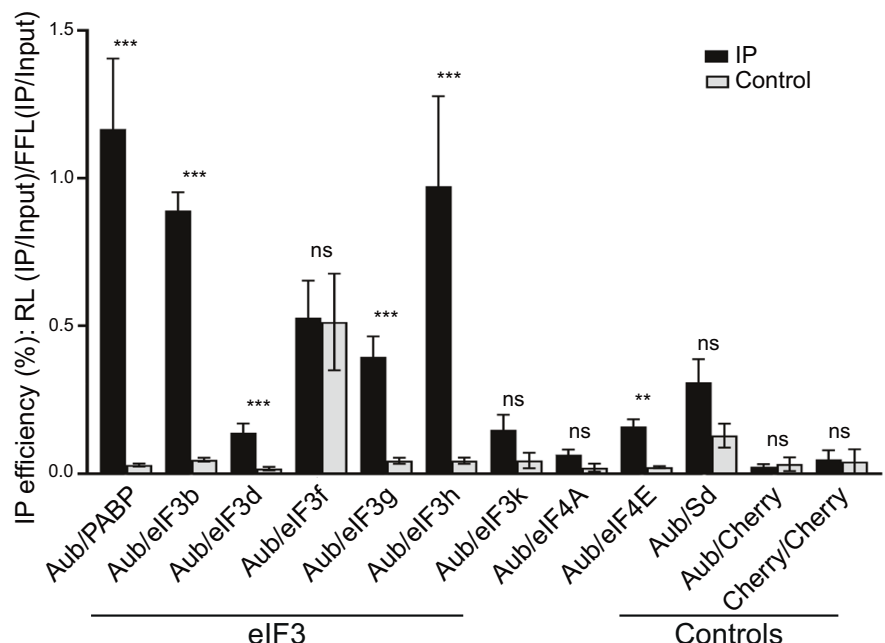


Fig. 3 Identification of Aub-interacting partners. a-d Volcano plots showing the mass spectrometry analysis of GFP-Aub immunoprecipitation from 0-2 h embryos. Embryos expressing cytoplasmic GFP were used as control. UASp-GFP-Aub nos-Gal4 embryos (a, b); osk ${ }^{54}$; UASp-GFP$A u b / n o s-G a l 4$ embryos $(\mathbf{c}, \mathbf{d})$. The analysis was based on four biological replicates. The red line indicates the significance threshold $(P=0.05)$. Known Aub interactors and RNA-binding proteins are indicated in red and purple, respectively (a, $\mathbf{c})$; translation initiation factors are indicated in blue (b, d). e GO analysis of proteins identified as Aub interactors by mass spectrometry. $\mathbf{f}$ Validation of Aub interactors using the LUMIER assay. Left: schematic representation of the assay (FFL Firefly luciferase; RL Renilla luciferase). Right: graph plotting the IP efficiency of the indicated proteins. The values are IP efficiencies of the coprecipitation of the RL fusion proteins (IP/Input) normalized by the IP/Input values for FLAG-FFL-Aub. Error bars represent SD. Stars indicate values significantly greater than six times the mean value obtained in the control IPs without anti-FLAG antibody (Control). Scalloped (Sd) and Cherry proteins were used as negative controls. ${ }^{* * *} P<0.001,{ }^{* *} P<0.01$, ns not significant, using the Z-test.

Nos protein levels in osk-OE embryos, consistent with a role of Aub and piRNAs in nos mRNA translation, independent of their role in transposable element regulation (Supplementary information, Fig. S1b).

Finally, most aub mutant embryos fail to develop, although they are fertilized. ${ }^{12,30}$ To address whether the lack of Nos protein in osk-OE; $a u b^{-1-}$ embryos could result from their arrest of embryonic development, we quantified Nos protein levels in osk-OE unfertilized eggs that are activated by egg laying but do not develop. Nos levels were similar in osk-OE unfertilized eggs and embryos, demonstrating that the defect in Nos protein synthesis in osk-OE; $a u b^{-1-}$ embryos did not result from their lack of embryonic development (Supplementary information, Fig. S1c).

Together, these results show that piRNA-guided Aub binding to nos mRNA plays a direct role in translational activation in the presence of Osk.

Ectopic expression of Osk leads to the formation of granules related to germ granules in the soma

In the germ plasm, Osk leads to the assembly of germ granules that are large ribonucleoprotein particles containing mRNAs required for germ cell specification and development. ${ }^{4,31}$ In addition to Osk, Aub is a core component of germ granules. ${ }^{32}$ We asked whether Osk ectopic expression in the somatic part of the embryo could lead to the formation of RNA granules related to germ granules, containing Aub and nos mRNA. Immunostaining of osk-OE embryos also expressing GFP-Aub revealed that Osk was present in the bulk of the embryo where it accumulated in cytoplasmic foci that became larger around nuclei (Fig. 2a). GFPAub was also present in cytoplasmic foci in the bulk of osk-OE embryos and in larger foci around nuclei. Small foci of either Osk or GFP-Aub were dispersed in the cytoplasm and did not colocalize. However, Osk and GFP-Aub colocalized in larger foci that surrounded nuclei, indicating a different composition of these large foci (Fig. 2a-a", e). smFISH of nos mRNA in embryos of the same genotype showed that nos mRNA accumulated in larger foci around nuclei where it colocalized with GFP-Aub (Fig. 2b-b", e). Strikingly, Nos protein also accumulated around nuclei and partially colocalized with GFP-Aub in large foci, suggesting that nos mRNA translation occurred in the vicinity of these granules (Fig. 2c-c", e). In contrast, in osk-OE embryos, Smg protein was present in foci that did not concentrate around nuclei and did not colocalize with large GFP-Aub foci (Fig. $2 d-d "$ ", e). This result was consistent with the reorganization of Smg into small foci in the germ plasm as compared to the somatic region in WT embryos, which suggested that Smg interaction with Osk did not take place within germ granules. ${ }^{13}$

We conclude that the presence of Osk in the somatic part of osk-OE embryos induces the formation of RNA granules that share functional similarities with germ granules, in which Aub and nos mRNA accumulate and at the proximity of which nos mRNA is translated.

Aub interacts with translation initiation factors

To further decipher the function of Aub, we identified Aub interactors in embryos. GFP-Aub was immunoprecipitated from
UASp-GFP-Aub nos-Gal4 $0-2 \mathrm{~h}$ embryos and the coprecipitated proteins were analyzed using mass spectrometry. Embryos expressing GFP alone were used as negative controls (Supplementary information, Fig. S2a). 107 proteins were significantly enriched in GFP-Aub immunoprecipitation (IP) $(P<0.05)$ (Supplementary information, Table S1). Known Aub interactors were identified, including Tudor (Tud) that is restricted to the germ plasm and required for Aub accumulation in the germ plasm, ${ }^{33,34}$ three components of the nos translation repressor complex, Trailer hitch (Tral), Belle (Bel) and Cup, ${ }^{35}$ and Capsuleen/PRMT5 (Csul), the methyltransferase responsible for Aub arginine dimethylation ${ }^{36}$ (Fig. 3a). Several RNAbinding proteins were also found in GFP-Aub IP (Fig. 3a). Importantly, six translation initiation factors were identified as Aub interactors, among which are PABP, three subunits of elF3 (elF3d, elF3k and elF3b), and elF4E, another component of nos translation repressor complex ${ }^{35}$ (Fig. 3 b). In addition, 48 ribosomal proteins coprecipitated with Aub (Supplementary information, Fig. S2b). Gene Ontology (GO) term enrichment analysis using FlyMine (http://www.flymine.org) identified "Translation" as the most enriched term among Aub interactors (Fig. 3e). We also analyzed Aub interactors in osk ${ }^{54}$ mutant embryos that do not form germ plasm, with the aim of identifying specific Aub interactors in the germ plasm, which might be lost in osk mutant embryos. However, mass spectrometry of GFP-Aub IP from osk ${ }^{54}$ mutant embryos identified a very similar set of proteins to that identified in osk $^{+}$embryos (Fig. 3c, d; Supplementary information, Fig. S2c, Table S1). These data suggested that Osk might not affect Aub interaction with most of its protein interactors, but rather their activity. Indeed, PABP and elF4E are found in the nos translational repressor complex, although they do not activate translation in this complex. ${ }^{35}$ elF3 subunits were found to be in complex with Aub in the absence of Osk, suggesting that they might also be present in the nos repressed mRNP. The presence of Osk, by remodeling the mRNP, would allow to switch on their activity in translational activation.

We used quantitative luminescence-based colP (LUMIER) assays to validate Aub interactions with translation initiation factors. ${ }^{37}$ Aub was fused to FLAG-tagged Firefly luciferase (FFL), whereas potential interactors were fused to Renilla luciferase (RL). Following transient expression in Drosophila S2R+cells, Aub was immunoprecipitated with anti-FLAG antibodies, or without antibodies as negative control, and interactor colP was quantified by recording Renilla and Firefly luciferase activities (Fig. 3f). PABP, four subunits of elF3 (elF3b, elF3d, elF3g and elF3h) among six tested subunits, and elF4E were found to significantly coprecipitate with Aub in these assays. Thus, although elF3k that was identified as an Aub interactor by mass spectrometry could not be confirmed with the LUMIER assay, elF3d and elF3b interaction with Aub was confirmed, and two other elF3 subunits, elF3g and elF3h were found to be in complex with Aub. Differences in the interaction between Aub and elF3 individual subunits between embryos and S2R+ cells likely resulted from differences in these two experimental systems.

These results reveal that Aub physically interacts with the translation machinery and are consistent with a direct function of Aub in translation regulation. 
a
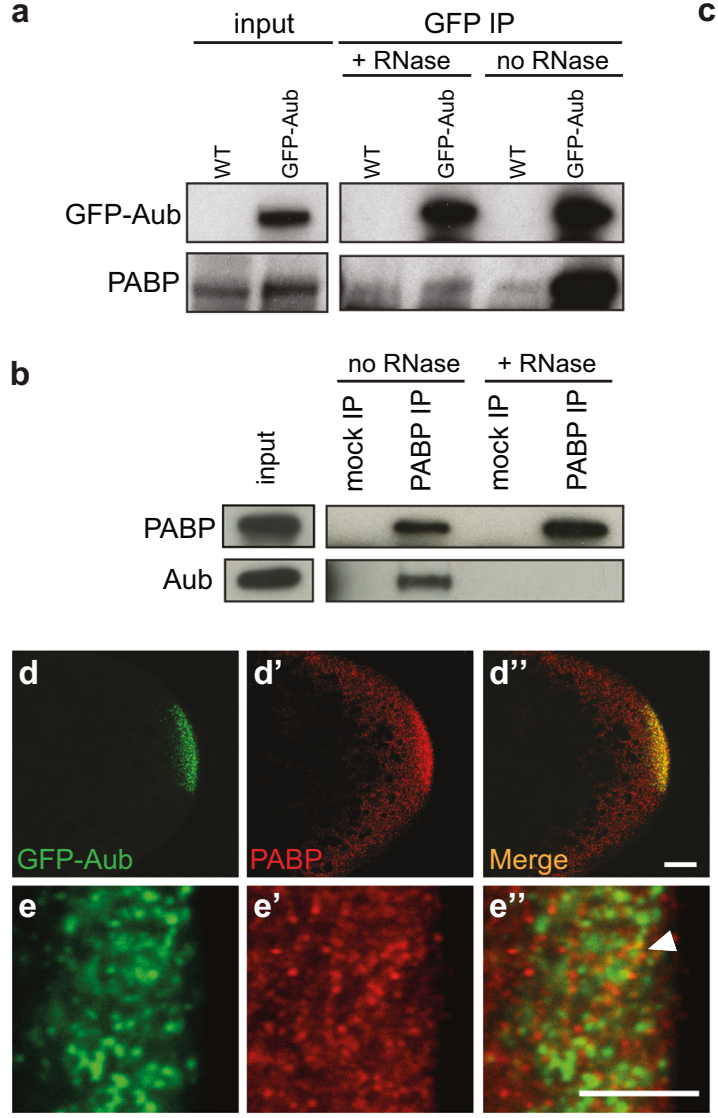

f

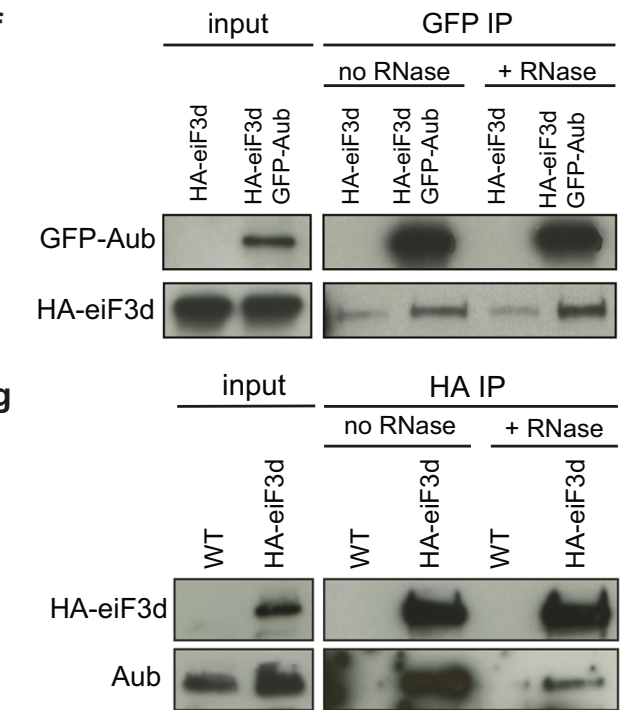

Aub interaction with PABP and elF3d

Because PABP and elF3d showed the strongest association with Aub in the mass spectrometry analysis, and have key roles in translation initiation, we further investigated their interaction with Aub. We used colP to address Aub physical interaction with PABP in embryos. PABP coprecipitated with GFP-Aub in $0-2 \mathrm{~h}$ embryos; however, this coprecipitation was strongly reduced in the presence of RNase (Fig. $4 \mathrm{a}$ ). In the reverse experiment, PABP was also able to coprecipitate Aub, but this coprecipitation was abolished in the presence of RNase (Fig. 4b). These results could indicate either that Aub and PABP did not interact directly and
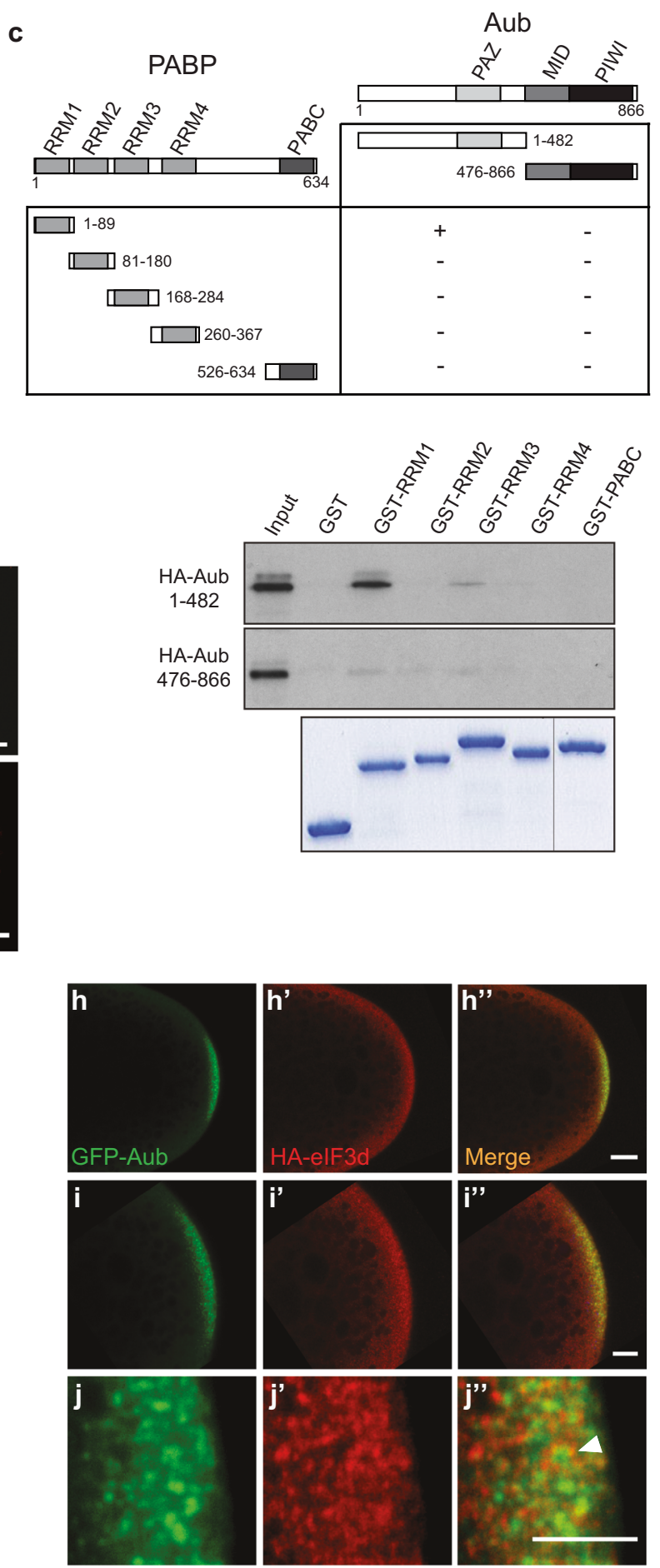

coprecipitated through their binding to the same mRNAs, or that Aub direct interaction with PABP was stabilized by mRNA in a tripartite association. To address this question, we analyzed direct interaction between Aub and PABP using GST pull-down assays. Aub has three domains characteristic of Argonaute proteins (PAZ, MID and PIWI) and was separated into two parts, Aub (1-482) that contains the N-terminal and PAZ domains, and Aub (476-866) that contains the MID and PIWI domains (Fig. 4c). PABP is composed of four RNA recognition motifs (RRM1-4), a proline-rich linker region and a PABP C-terminal (PABC) domain. ${ }^{38}$ Each RRM and the PABC domain were fused 
Fig. 4 Aub physical interaction with PABP and elF3d. a, b ColP of PABP with GFP-Aub (a) and of Aub with PABP (b) in 0-2 $\mathrm{h}$ embryos. WT (mock IP) or nos-Gal4 UASp-GFP-Aub (GFP IP) embryo extracts were immunoprecipitated with anti-GFP, either in the absence or the presence of RNase A. Western blots were revealed with anti-GFP and anti-PABP (a). WT embryo extracts were immunoprecipitated with anti-PABP (PABP IP) or rabbit serum (mock IP), either in the absence or the presence of RNase A. Western blots were revealed with anti-PABP and anti-Aub (b). Inputs are extracts before IP in a and $\mathbf{b}$. c GST pull-down assays between GST-PABP and HA-Aub. Constructs and interactions are shown in the table. HA-tagged Aub fragments were revealed using western blot with anti-HA. Inputs correspond to $1 / 10$ of in vitro synthesized HA-Aub fragments before pull-down. GST alone was used as negative control. GST and GST-recombinant proteins used in each pull-down are shown in the bottom gel. d-e" Immunostaining of UASp-GFP-Aub nos-Gal4 embryos with anti-GFP (green) to visualize Aub and anti-PABP (red). Posterior of embryos are shown. Higher magnification showing the distribution of Aub-containing germ granules and PABP foci (e-e"). Colocalization and overlap between Aub and PABP staining are quantified in Supplementary information, Fig. S3a. The white arrowhead shows PABP foci surrounding a germ granule. Scale bars, $20 \mu \mathrm{m}$ in $\mathbf{d}$ and $5 \mu \mathrm{m}$ in e. f, $\mathbf{g}$ ColP of HA-elF3d with GFP-Aub (f) and of Aub with HA-elF3d (g) in 0-2 $\mathrm{h}$ embryos. UASp-HA-elF3d/+; nos-Gal4/+ (mock IP) or UASp-HA-elF3d/+; UASp-GFP-Aub nos-Gal4/+ (GFP IP) embryo extracts were immunoprecipitated with anti-GFP, either in the absence or the presence of RNase A. Western blots were revealed with anti-GFP and antiHA (f). WT (mock IP) or UASp-HA-elF3d/+; nos-Gal4/+ (HA IP) embryo extracts were immunoprecipitated with anti-HA, either in the absence or the presence of RNase A. Western blots were revealed with anti-HA and anti-Aub (g). Inputs are extracts before IP in $\mathbf{f}$ and $\mathbf{g}$. $\mathbf{h}-\mathbf{j}$ " Immunostaining of UASp-HA-elF3d/+; UASp-GFP-Aub nos-Gal4/+ embryos with anti-GFP (green) to visualize Aub and anti-HA (red) to visualize elF3d. Posterior of embryos are shown. Higher magnification showing the slight accumulation of HA-elF3d at the posterior pole (i-i"), and the distribution of Aub-containing germ granules and elF3d foci ( $\mathbf{j}-\mathbf{j}$ "). Colocalization and overlap between Aub and elF3d staining are quantified in Supplementary information, Fig. S3d. The white arrowhead shows elF3d foci surrounding a germ granule. Scale bars, $20 \mu \mathrm{m}$ in $\mathbf{h}, 10 \mu \mathrm{m}$ in $\mathbf{i}$ and $5 \mu \mathrm{m}$ in $\mathbf{j}$.

separately to GST. In vitro-translated HA-tagged Aub(1-482) bound to recombinant GST-RRM1, but not to the other PABP domains fused to GST or GST alone. In addition, HA-Aub (476-866) did not bind to any PABP domain (Fig. 4c). These data revealed direct interaction between RRM1 of PABP and the $\mathrm{N}$ terminal half of Aub. They were consistent with the model in which Aub directly interacted with PABP and mRNA stabilized this interaction in embryos.

We then analyzed potential colocalization of Aub and PABP. Coimmunostaining of GFP-Aub-expressing embryos with anti-PABP and anti-GFP antibodies showed that PABP was distributed in the whole embryo and specifically accumulated in the germ plasm (Fig. $\left.4 d-d^{\prime \prime}\right)$. Strikingly, PABP was present in foci, and in the germ plasm a large proportion of Aub-containing germ granules $(79.4 \%$, Supplementary information, Fig. S3a) either colocalized with, or were in close proximity to and surrounded by PABP foci, with a partial overlap of both proteins (Fig. 4e-e"; Supplementary information, Fig. S3a).

elF3 is composed of twelve subunits and one associated factor, and coordinates several steps of translation initiation. ${ }^{39}$ Interestingly, in addition to this role in basal translation, elF3 plays regulatory roles in the translation of specific mRNAs. elF3d appears to be a major actor in elF3 regulatory functions, either through its binding to $5^{\prime}$ UTR of specific mRNAs, leading to capindependent translation, or directly through its interaction with the cap structure. ${ }^{40,41}$ Aub interaction with elF3d was analyzed in embryos using colP. GFP-Aub was able to coprecipitate HAelF3d in $0-2 \mathrm{~h}$ embryos, and this coprecipitation was maintained in the presence of RNase (Fig. 4f). Conversely, HA-elF3d was able to coprecipitate Aub in 0-2 h embryos, and although less efficient, this coprecipitation remained in the presence of RNase (Fig. 4g). Colocalization of Aub and elF3d was analyzed in embryos expressing both GFP-Aub and HA-elF3d. elF3d was present in the whole embryo with a slight accumulation in the germ plasm (Fig. 4h-i"; Supplementary information, Fig. S3b, c). Similarly to PABP, elF3d formed foci, and in the germ plasm most Aub-containing germ granules (83.2\%, Supplementary information, Fig. S3d) colocalized with or were surrounded by elF3d foci, with a partial colocalization of both proteins at the edge of the granules (Fig. 4j-j"; Supplementary information, Fig. S3d).

Taken together, these results show that Aub is in complex with the translation initiation factors PABP and elF3d. In the germ plasm, PABP and elF3d have a specific organization around germ granules and colocalize with Aub at the periphery of the granules, suggesting that translation might take place at the edge of germ granules.

\section{Mechanism of Aub-dependent translational activation}

Aub association with translation initiation factors suggested that Aub might activate nos mRNA translation at the level of initiation. We directly addressed this question using polysome profiling in which mRNA-protein complexes are separated by fractionation through linear sucrose gradients. ${ }^{42}$ mRNA localization within the sucrose gradient reflects its translation status: migration in the light RNP or monosomal fractions of the gradient indicates a lack of translation, whereas migration in the heavy polysomal fractions indicates active translation. Polysome profiling was performed with $0-2 \mathrm{~h}$ WT, osk-OE and osk-OE; $a u b^{-1-}$ embryos. The abundance of polysomes was reduced in osk-OE embryos compared to WT, indicating that ectopic expression of Osk in the whole embryo affected basal translation (Fig. 5a). In contrast, polysome abundance was partially restored in osk-OE; aub ${ }^{-1-}$ embryos, revealing that translation was active in these embryos (Fig. 5a). Thus, the level of basal translation was affected oppositely to the level of Nos protein. This is consistent with Aub being involved in a regulatory mode of translation occurring on specific mRNAs. To confirm this point, we used smg mRNA as a control, since it is highly translated in the whole embryo upon egg activation. ${ }^{43,44} \mathrm{Smg}$ protein levels were not decreased in osk-OE; $a u b^{-1-}$ embryos compared to osk-OE embryos, confirming the specificity of Aub-dependent translational activation (Supplementary information, Fig. S4a). Western blot analysis of the gradient fractions revealed co-sedimentation of Aub with actively translating mRNAs in the heavy polysomal fractions, and the presence of PABP in these fractions (Fig. 5b, c). To confirm Aub association with actively translating mRNAs, we treated embryo lysates with puromycin that causes premature termination of elongating ribosomes. Puromycin treatment efficiency was validated by the complete disassembly of polysomes visualized by absorbance measurement of OD at $254 \mathrm{~nm}$, and the shift of ribosomal proteins to monosomal and lighter fractions containing $60 \mathrm{~S}$ and $40 \mathrm{~S}$ ribosomal subunits (Fig. 5b, c). Aub shifted to the light mRNP fractions in the presence of puromycin, indicating its bona fide association with translating mRNAs. In contrast, although PABP was shifted towards lighter fractions of the gradients in the presence of puromycin, a certain amount remained present in most fractions, suggesting the presence of heavy RNA complexes containing PABP in Drosophila embryos (Fig. 5c). This is consistent with the presence of mRNAs in heavy fractions of sucrose gradients independently of translation, in polysome gradients from early embryos. ${ }^{45}$ We then quantified mRNA through polysome gradients using RT-qPCR. nos mRNA was mostly present in initiation and light polysomal fractions in WT embryos, in agreement with a low amount of nos mRNA being actively 


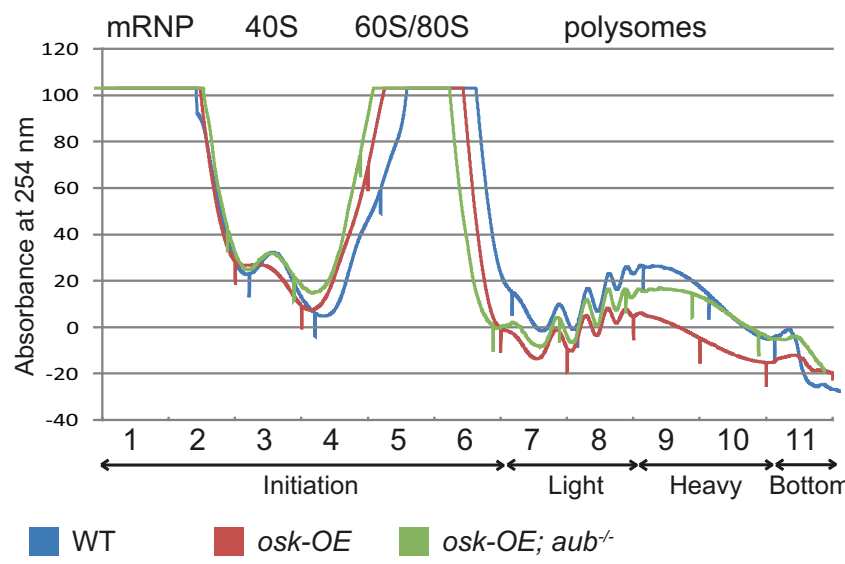

b

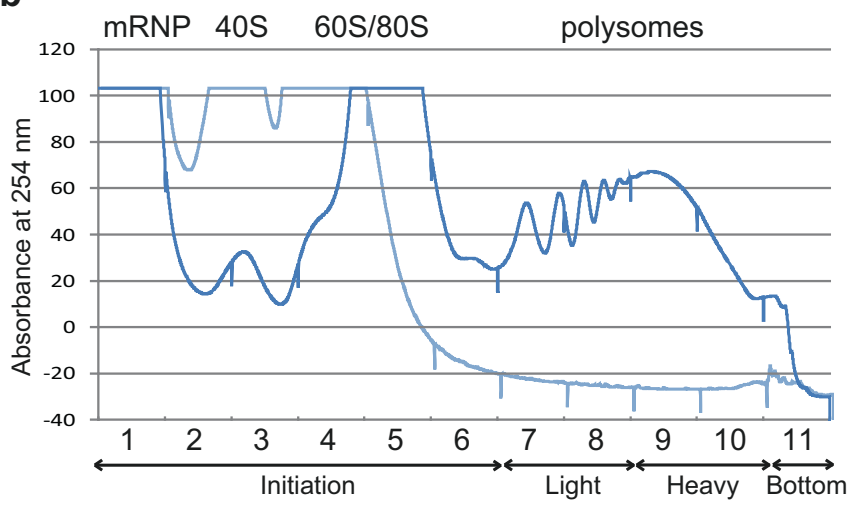

d

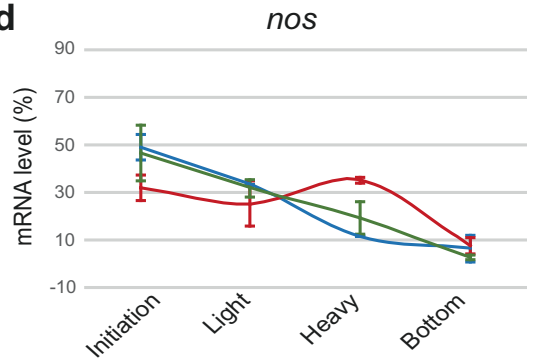

e

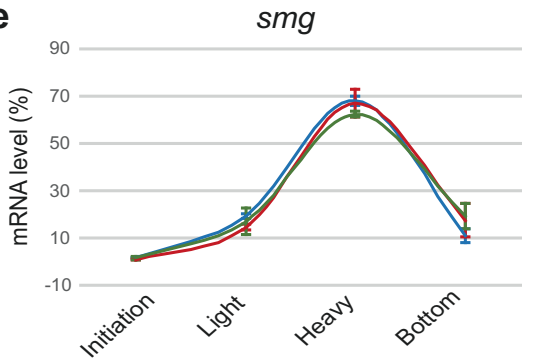

f

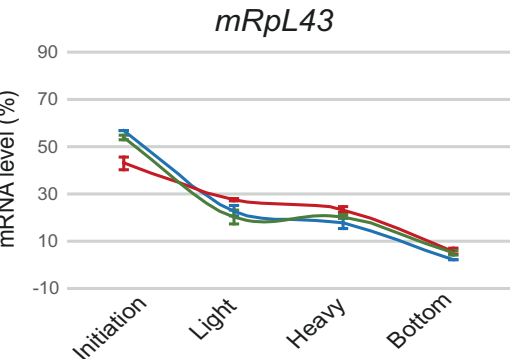

WT

WT + puromycin

C

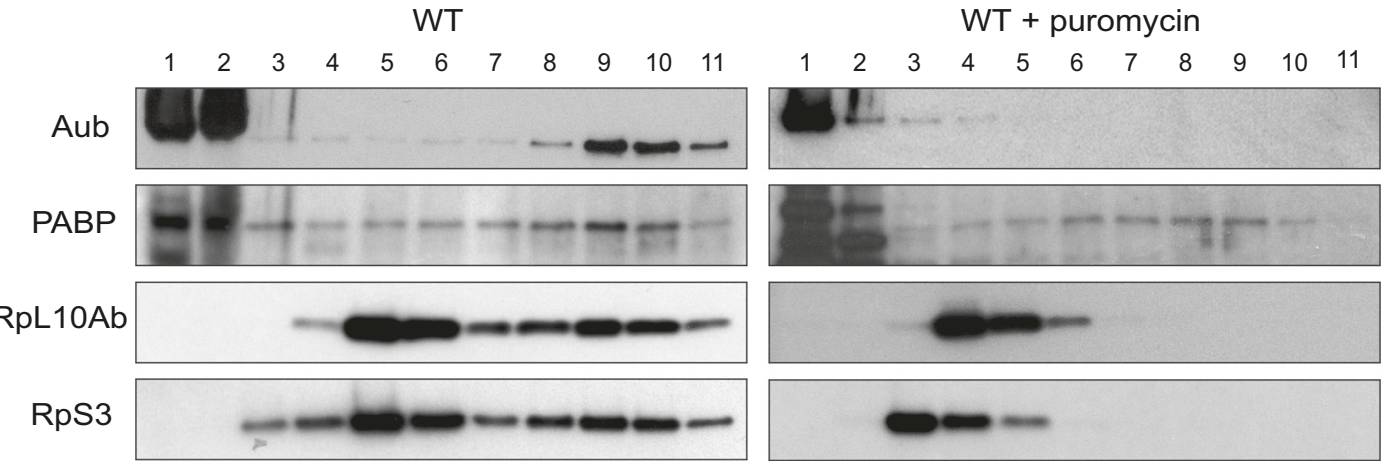

Fig. 5 Aub acts at the level of translation initiation. a Profile of absorbance at $254 \mathrm{~nm}$ for $0-2 \mathrm{~h}$ WT (blue), osk-OE (red) and osk-OE; aub ${ }^{-/}$ (green) embryo extracts fractionated into $10 \%-50 \%$ sucrose gradients. Complete genotypes are as in Fig. 1. Fractions were pooled as indicated on the graph into: initiation (fractions 1-6), light polysomes (fractions 7 and 8), heavy polysomes (fractions 9 and 10) and bottom (fraction 11). b Profile of absorbance at $254 \mathrm{~nm}$ for 0-2 $\mathrm{h}$ WT embryos treated (light blue), or not (dark blue) with puromycin, fractionated into $10 \%-50 \%$ sucrose gradients. c Western blot showing the distribution of Aub and PABP through the gradient from WT embryos treated or not with puromycin. Two ribosomal proteins, RpL10Ab (60S ribosome subunit) and RpS3 (40S ribosome subunit) were used to record puromycin treatment efficacy. d-f Quantification of nos (d), smg (e) and mRpL43 (f) mRNAs using RT-qPCR in the different fractions of the gradients for WT (blue), osk-OE (red) and osk-OE; aub ${ }^{-/-}$(green) embryos. mRNA levels are indicated in percentage of total mRNA in all the fractions. Mean of two biological replicates, quantified in triplicate. Error bars represent SEM. smg and mRpL43 were used as control mRNAs.

translated (Fig. 5d). In osk-OE embryos, the level of nos mRNA decreased in the initiation fractions whereas it increased in the heavy polysomal fractions, consistent with the 2 -fold increase of Nos protein levels in these embryos (Figs. 1a, b, 5d). Quantification of nos mRNA through the gradient in the presence of puromycin confirmed that the pool of nos present in the heavy fractions was indeed associated with actively translating polysomes (Supplementary information, Fig. S4b-d). Interestingly, in osk-OE; aub ${ }^{-1-}$ embryos, the distribution of nos MRNA was similar to that in WT embryos, with higher amounts of mRNA in initiation fractions and lower amounts in heavy polysomal fractions (Fig. 5d). These results suggested the role of Aub at the initiation step of translation. To 
a

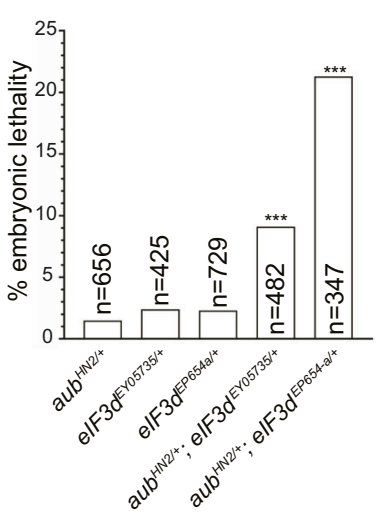

b

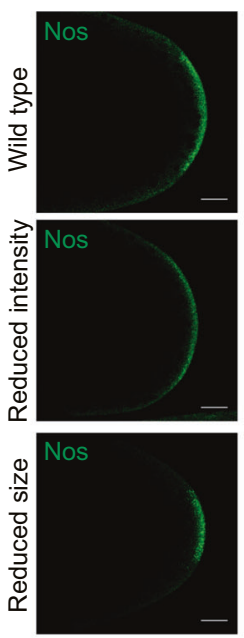

C

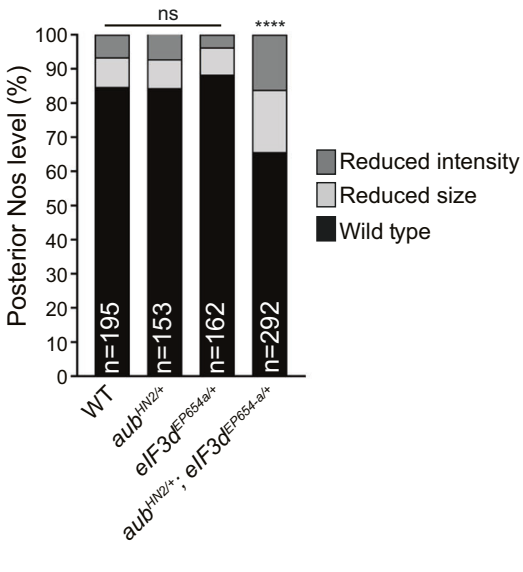

d

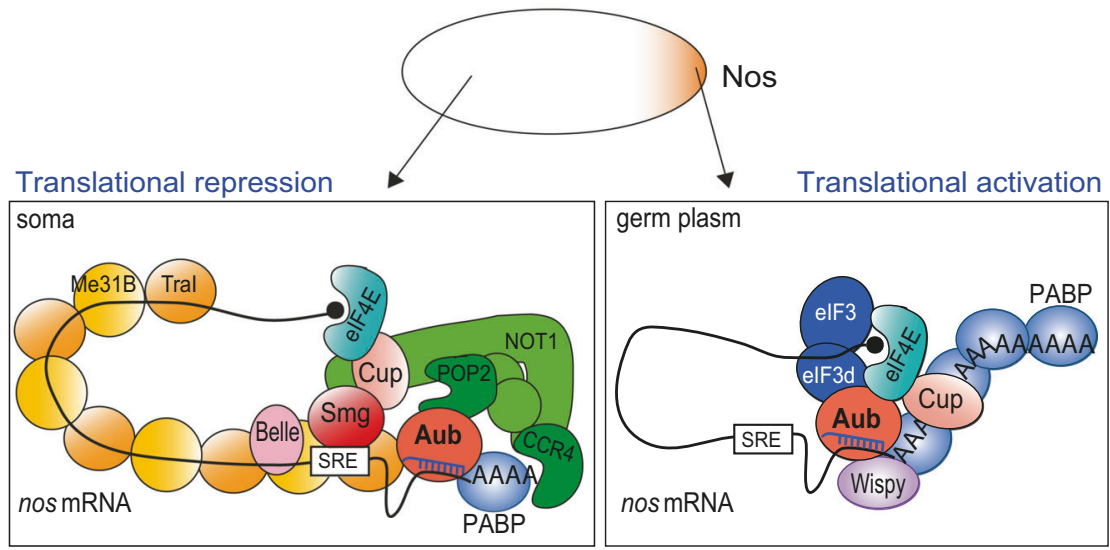

Fig. 6 Aub and elF3d functionally interact for nos mRNA translation. a Percentage of embryonic lethality of single or double aub and elF3d heterozygous mutants. The genotypes are indicated. ${ }^{* *} P<0.001$, using the $\chi^{2}$ test. $\mathbf{b}$ Immunostaining of single and double aub and elF3d heterozygous mutant embryos with anti-Nos antibody. Posterior of embryos with the three types of staining: wild type, reduced size or reduced intensity, are shown. Scale bars, $20 \mu \mathrm{m}$. c Quantification of posterior staining shown in b using the ImageJ software. For each genotype, the percentage of embryos with each staining category was recorded. ${ }^{* * *} P<0.0001$, ns not significant, using the $\chi^{2}$ test. d Model of Aub-dependent translational activation. In the somatic part of the embryo, nos mRNA translation is repressed by two mechanisms: a capdependent mechanism that involves Cup binding to elF4E, and a cap-independent mechanism that involves the coating of the mRNA by Me31B and Tral. Both mechanisms might depend on the CCR4-NOT complex recruited by Smg and Aub. In the germ plasm, Smg binding to Osk precludes its interaction with nos mRNA, leading to depletion of CCR4-NOT and remodeling of the mRNP. This would lead to the dissociation of Me31B/Tral from the mRNA. Aub interaction with PABP and elF3 subunits would allow unconventional translation, bypassing eIF4E requirement. The recruitment of Wispy poly $(A)$ polymerase by Aub leading to polyadenylation is likely to also contribute to translation activation. Note that elF3 might be present in the repressor complex in the soma, since elF3 was found as Aub interactor in osk mutant embryos; however, its activity in translation activation would be repressed.

further confirm the role of Aub in translational activation of specific mRNAs, we quantified smg and $m R p L 43$ mRNAs through the polysome gradients. Consistent with smg active translation in early embryos, most smg mRNA was present in heavy polysomal fractions, and this profile was not affected in osk-OE and osk-OE; $a u b^{-1-}$ embryos, indicating that $s m g$ translation was independent of both Osk and Aub (Fig. 5e). mRpL43 was used as a control mRNA that is not bound by Aub ${ }^{12}$ and similarly, its distribution through the gradient was not strongly affected in osk-OE and osk$O E ; a u b^{-1-}$ embryos (Fig. 5f).

These results show that Aub plays a role in the translation of specific mRNAs and are consistent with Aub acting at the level of translation initiation.

elF3d plays a role in Aub-dependent translational activation To address the biological relevance of Aub/elF3d physical interaction, we analyzed the effect of the concomitant reduction of $a u b$ and elF3d gene dosage by half. Although single $a u b$ or eif3d heterozygous mutant embryos showed a low level of lethality $(2 \%-3 \%)$, embryonic lethality significantly increased up to $21 \%$ in double heterozygous mutants, suggesting that Aub and elF3d act together in embryonic development (Fig. 6a). nos mRNA translation was then recorded in these embryos using immunostaining. The Nos protein level visualized by immunofluorescence at the posterior cortex was quantified. In WT, $85 \%$ of embryos showed a full accumulation of Nos protein at the posterior pole, whereas $15 \%$ had a reduced accumulation (Fig. 6b, c). Nos accumulation in heterozygous aub or elF3d mutant embryos was similar to that in WT. In contrast, in $a u b^{-1+}$; elF3d $d^{-1+}$ double heterozygous mutants, the percentage of embryos with reduced Nos accumulation significantly increased to $34 \%$ (Fig. 6b, c; Supplementary information, Fig. S5a). This reduction of Nos accumulation in double heterozygous mutants did not correlate with reduced Osk accumulation or reduced nos mRNA localization at the posterior pole (Supplementary information, Fig. S5b-e), indicating a direct defect in nos mRNA translation. We conclude that Aub/elF3d physical interaction is required for nos mRNA translational activation. 


\section{DISCUSSION}

Several studies have reported the role of PIWI proteins in cellular mRNA regulation at the level of stability. piRNA-dependent binding of mRNAs by PIWI proteins leads to their decay in different biological systems. ${ }^{16}$ In addition, in Drosophila embryos, mRNA binding by the PIWI protein Aub also leads to their stabilization in a spatially regulated manner. ${ }^{13}$ Here, we report a novel function of Aub in direct translational control of mRNAs. Using nos mRNA as a paradigm, we show that Aub is required for nos mRNA translation. Nos protein levels are also strongly reduced in armi mutant, in which piRNA biogenesis is massively affected, ${ }^{27}$ suggesting that Aub loading with piRNAs is necessary for its function in translational activation. Consistent with this, we find that deletion of two piRNA target sites in nos mRNA decreases its translation. Importantly, Nos levels are not affected in a panx mutant background. Panx is a piRNA factor required for transcriptional repression of transposable elements, but has no function in piRNA biogenesis. ${ }^{28,29}$ In addition, as is the case for aub and armi mutants, panx mutant embryos do not develop. ${ }^{28,29}$ Finally, Nos levels are similar in unfertilized eggs and embryos overexpressing Osk, demonstrating that Nos protein synthesis is independent of embryonic development. Together, these results strongly argue for a direct role of Aub and piRNAs in nos mRNA translational control, independently of their role in transposable element regulation or developmental defects in piRNA pathway mutants.

Mass spectrometry analysis of Aub interactors points to a strong link with the translation machinery. In addition, polysome gradient analyses reveal Aub association with actively translated mRNAs in polysomal fractions. A link has been reported previously between the PIWI proteins Miwi and Mili and the translation machinery in mouse testes, where Miwi and Mili were found to associate with the cap-binding complex. ${ }^{46,47}$ However, the role of Miwi and Mili in translational control has not been characterized. We now decipher the molecular mechanisms of Aub function in translational activation of germ cell mRNAs in the Drosophila embryo. We demonstrate a physical interaction between Aub and the translation initiation factors PABP, elF4E and subunits of the elF3 complex. These interactions are in agreement with polysome gradient analyses in WT and aub mutant backgrounds that indicate a role of Aub in translation initiation.

Recent data have identified specific roles of elF3 in the regulation of translation. elF3 is the most elaborate of translation initiation factors containing twelve subunits and an associated factor, elF3j. This complex promotes all steps of translational initiation and does so in part through direct association with other translation initiation factors, contributing to their functional conformations on the small ribosomal subunit surface. ${ }^{39}$ In addition to this role in basal translation, the elF3a, b, $d$ and $g$ subunits were shown to directly bind $5^{\prime}$ UTR of specific mRNAs, leading to cap-dependent translation activation or repression. ${ }^{40}$ The elF3d subunit that attaches to the edge of the complex appears to play an especially important role in various modes of elF3-dependent translational control: (1) elF3d is involved in the translational repression of Drosophila sex-lethal mRNA through binding to its $5^{\prime} U T R{ }^{48}$ (2) elF3d was reported to directly bind the cap structure of specific mRNAs in mammalian cells, thus bypassing the requirement of elF4E binding to the cap for translation initiation. ${ }^{41}$ (3) In the same line, elF3d was involved in cap-dependent translational activation of specific mRNAs for neuronal remodeling in Drosophila larvae, in a context where elF4E is blocked by $4 \mathrm{E}$-binding protein $(4 \mathrm{E}-\mathrm{BP}){ }^{49}$ Other studies have reported the role of elF3 in promoting cap-independent translation, thus highlighting elF3 functional versatility in the control of translation. elF3 was shown to directly bind methylated adenosine $\mathrm{m}^{6} \mathrm{~A}$, in mRNA $5^{\prime} \mathrm{UTR}$ to induce cap-independent translation under stress conditions. ${ }^{50}$ Furthermore, PABP bound to the $\operatorname{poly}(A)$ tail was also shown to cooperate with elF3 for its binding to mRNA 5'UTR triggering cap-independent translation. ${ }^{51}$
Here, we described a new mode of elF3-dependent translational activation through its recruitment by the PIWI protein Aub. Based on previous information on the nos translation repressor complex and data presented here on translational activation, we propose the following model (Fig. $6 \mathrm{~d}$ ). nos mRNA translation is repressed in the somatic part of the embryo by two mechanisms. ${ }^{11,35}$ First, the 4E-BP protein Cup in complex with Smg binds to elF4E and prevents elF4G recruitment and cap-dependent translation. ${ }^{11,52}$ The detailed mechanism of Cup recruitment to the repressor complex has not been clarified, but Cup was shown to directly associate with the Not1 subunit of the CCR4-NOT complex and this interaction might stabilize Cup association with elF4E. ${ }^{53}$ CCR4NOT itself is recruited to nos mRNA by Smg and Aub. ${ }^{9,18}$ Second, two translational repressors, the RNA helicase Me31B (Drosophila DDX6) and its partner Tral coat the length of nos mRNA and prevent translation through a cap-independent mechanism. ${ }^{35}$ Again the mode of Me31B/Tral specific recruitment to nos mRNA has not been determined, but the CCR4-NOT complex might also be involved since DDX6 directly binds the Not1 subunit of CCR4NOT. ${ }^{54,55}$ Aub coprecipitation with components of the nos translational repressor complex is consistent with its association with the CCR4-NOT complex in the soma ${ }^{18}$ and suggests that Aub might be involved in translational repression, in addition to mRNA decay. In the germ plasm, Osk interaction with Smg prevents Smg binding to nos $\mathrm{mRNA}^{9}$ and this contributes to CCR4-NOT displacement from the mRNP complex. Consistent with this, CCR4 is depleted in the germ plasm. ${ }^{13}$ The lack of CCR4-NOT on nos mRNA might preclude the recruitment of Me31B/Tral and relieve the cap-independent mechanism of translational repression (Fig. 6d). We find that Aub physically interacts with PABP and several subunits of elF3. We propose that these associations would lead to translational activation independently of elF4E through binding of elF3 to nos 5'UTR, followed by direct recruitment of the $40 \mathrm{~S}$ ribosome by elF3 and PABP, as previously reported for translation of XIAP mRNA. ${ }^{51}$ Alternatively, elF3 might act through direct binding of elF3d to the cap structure; however, we do not favor this hypothesis. Indeed, if elF3d interaction with the cap was involved, overexpression of the point mutant elF3d $d^{\text {helix } 11}$ that is unable to bind the cap, ${ }^{41}$ would be expected to induce negative dominant defects, due to the lack of translation mediated by this interaction. ${ }^{49}$ However, overexpression of elF3d ${ }^{\text {helix11 }}$ with the nosGal4 driver did not induce any defects in embryonic development or Nos protein synthesis (Supplementary information, Fig. S6).

Germ granules coordinate germ cell mRNA regulation with piRNA inheritance through the role of PIWI proteins in both processes. Recent studies in C. elegans have shown that piRNA/ PRG1-dependent mRNA accumulation in germ granules prevent their silencing, strengthening the function of piRNAs in germ granules for mRNA storage and surveillance. ${ }^{56,57}$ In Drosophila, Aub mediates the link between piRNAs and mRNA regulation in germ granules since Aub localization to germ granules depends on its loading with piRNAs ${ }^{12}$ and Aub/piRNAs play a general role in the localization and stabilization of germ cell mRNAs in germ granules. ${ }^{13,19}$ How do germ granules accommodate translational control has remained more elusive. In Drosophila embryos, germ granules contain mRNAs that are translated sequentially. ${ }^{58}$ We demonstrate a direct role of Aub in translational activation. Strikingly, PABP and elF3d tend to colocalize with Aub at the periphery of germ granules. This is reminiscent of a study analyzing translational control in relation to RNA granules in Drosophila oocytes, in which translational repressors such as Me31B were found to concentrate in the granule core with repressed mRNAs, whereas the translational activator Orb was localized at the edge of the granules where mRNAs docked for translation. ${ }^{59}$ Similarly, germ granules in embryos might be partitioned into functional subdomains involved in various steps of mRNA regulation, including storage (in an internal region of granules) and translational activation (at the granule periphery). 
Our work reveals the central role of Aub in activation of translation. Future studies will undoubtedly address the complexity of mRNA regulation by PIWI proteins in relation with germ granules.

While this manuscript was under review, a role of Miwi and piRNAs in translational activation during mouse spermiogenesis has been demonstrated. ${ }^{60}$ Miwi was shown to be in complex with PABP and several subunits of elF3 for its function in translational activation, which is required for spermatid development. This reveals a striking evolutionary conservation of PIWI protein function in translational control for key developmental processes.

\section{MATERIALS AND METHODS}

\section{Drosophila lines}

$w^{1118}$ was used as a control. Mutant alleles and transgenic lines were $a u b^{Q C 42} \mathrm{cn}^{1} b w^{1} / \mathrm{CyO}$ and $a u b^{H N 2} \mathrm{cn}^{7} b w^{1} / C y O_{1}^{61}$ nos-Gal4VP16, ${ }^{62}$ UASp-osk-K10, ${ }^{25}$ panx $^{M 1}$ and panx ${ }^{M 429}$ armi $^{1}{ }^{163}$ armi $^{72.1}{ }^{164}$ $\operatorname{nos}^{B N \times 65}$ nos(Dpiroo $\triangle$ pi412); nos ${ }^{B N} / T M 3$ Sb, ${ }^{13}$ w; osk ${ }^{54}$ nos-Gal4VP16/TM3 Sb and yw; osk ${ }^{54}$ e UASp-GFP-Aub/TM3 Sb, ${ }^{12}$ UASp-GFP$A u b{ }^{66}$ UAS-GFP cytoplasmic (gift from J.M. Dura), elF3d ${ }^{\text {EY05735 }}$ (\#20072) and elF3 $d^{E P-654 a}$ (\#43437) (Bloomington Drosophila Stock

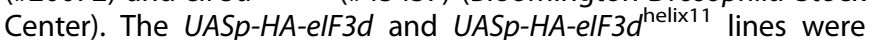
generated in this study by insertion of $\mathrm{PhiC} 31$ recombination into attP40 site (BestGene). The genotypes of embryos (aged $0-2 \mathrm{~h}$ ) indicated throughout were the genotypes of mothers. Females of the indicated genotypes were crossed with WT males.

$\mathrm{S} 2 \mathrm{R}+$ cells

$\mathrm{S} 2 \mathrm{R}+$ cells (Gift from G. Cavalli) were cultivated at $25^{\circ} \mathrm{C}$ in Schneider medium complemented with $10 \%$ fetal bovine serum (Gibco) and 1\% penicillin-streptomycin (Gibco).

\section{Immunostaining and image analysis}

0-2 $\mathrm{h}$ embryos were collected in a basket from plates, washed in tap water and dechorionated using commercial bleach for $2 \mathrm{~min}$, rinsed and dried. Embryos were then fixed at the interface of a $1: 1$ solution of $36 \%$ formaldehyde: $100 \%$ heptane for $5 \mathrm{~min}$, followed by $100 \%$ methanol devitellinization. Embryos were rehydrated, blocked in 1\% BSA for $1 \mathrm{~h}$ and incubated overnight with primary antibodies. Secondary antibody incubation, after washes in PBS, $0.1 \%$ Tween, was performed for $1 \mathrm{~h}$ at room temperature. Embryos were mounted in Vectashield (Vector Laboratories) for imaging. Antibodies used: rabbit anti-Osk (1:1000, gift from $P$. Lasko), rabbit anti-Nos (1:1000, gift from $A$. Nakamura), rabbit anti$\operatorname{PABP}\left(1: 500\right.$, gift from A. Vincent), rabbit anti-Smg (1:2000), ${ }^{67}$ mouse anti-HA (1:2000, ascites produced from clone 12CA5), rabbit anti-GFP (1:1000, Invitrogen), mouse anti-GFP (1:1000, Roche), goat anti-mouse IgG Cy3 (1:1000, Jackson ImmunoResearch), goat anti-rabbit IgG Alexa-488 (1:800, Invitrogen) and donkey anti-rabbit Cy3 (1:1000, Jackson ImmunoResearch). Microscopy was performed using a Leica SP8 confocal scanning microscope. Data were processed and analyzed using the ImageJ software.

\section{smFISH}

Dechorionated embryos were fixed at the interface of a $1: 1$ solution of $10 \%$ formaldehyde: $100 \%$ heptane for $20 \mathrm{~min}$, followed by $100 \%$ methanol devitellinization. After permeabilization in ethanol, embryos were washed 4 times for 15 min in PBT and then once for 20 min in Wash Buffer (10\% 20x SCC, 10\% formamide). They were then incubated overnight at $37^{\circ} \mathrm{C}$ in Hybridization Buffer (10\% formamide, 10\% 20× SSC, $400 \mu \mathrm{g} / \mathrm{mL}$ tRNA, 5\% Dextran sulfate, 1\% VRC (Vanadyl Ribonucleoside Complexes, Sigma)) with anti-nos probes (Supplementary information, Table S2) coupled to CAL Fluor Red 590 (Stellaris). Embryos were washed in Wash Buffer at $37^{\circ} \mathrm{C}$ and then in $2 \times \mathrm{SCC}, 0.1 \%$ Tween at room temperature before mounting (Pro-Long Gold antifade reagent, Invitrogen). Microscopy was performed using a Leica SP8 confocal scanning microscope. Data were processed and analyzed using the ImageJ software.

\section{RNA extraction and RT-qPCR}

Total RNA was prepared from 30 embryos using Trizol (Invitrogen) following recommendations from the manufacturer. For RT-qPCR, $1 \mu \mathrm{g}$ of total RNA was reverse transcribed using Superscript III (Invitrogen) and random hexamers. Quantitative PCR (qPCR) was performed on a LightCycler LC480 (Roche) with Lightcycler 480 SYBR green master (Roche) and primers are listed in Supplementary information, Table S3. Quantifications were performed in triplicate.

\section{Coimmunoprecipitation and western blot}

GFP immunoprecipitations for mass spectrometry were performed as follows: $0.5 \mathrm{~g}$ of $0-2 \mathrm{~h}$-dechorionated embryos were crushed in DXB buffer ( $25 \mathrm{mM}$ HEPES, $250 \mathrm{mM}$ sucrose, $1 \mathrm{mM} \mathrm{MgCl}$, $1 \mathrm{mM}$ DTT, $150 \mathrm{mM} \mathrm{NaCl}$, protease inhibitor) with $0.1 \%$ Triton X-100 and RNasin and incubated on ice for 30 min. Lysates were centrifuged for $10 \mathrm{~min}$ and the supernatant was transferred to a new tube. Lysates were incubated on equilibrated GFP-trap beads (Chromotek) overnight at $4{ }^{\circ} \mathrm{C}$ on a wheel. Beads were washed seven times in DXB buffer complemented with $1 \%$ Triton X-100 and RNasin. Beads were suspended in $2 \times$ NuPAGE Blue supplemented with $50 \mathrm{mM}$ DTT and incubated for $10 \mathrm{~min}$ at $95^{\circ} \mathrm{C}$. The quality of the samples was assessed by silver staining (SilverQuest, Invitrogen). For colP experiments, $0.15-0.18 \mathrm{mg}$ of $0-2 \mathrm{~h}$ embryos were crushed in IP buffer (20 mM Tris, pH 7.5, $150 \mathrm{mM} \mathrm{NaCl}, 0.2 \%$ NP40, $1.5 \mathrm{mM}$ DTT, $10 \mathrm{mM}$ EGTA, protease inhibitor) with either $40 \mathrm{U} /$ $\mu \mathrm{L}$ RNase $\mathrm{A}$ or $100 \mathrm{U} / \mu \mathrm{L}$ RNase inhibitor. Extracts were centrifuged at $10,000 \times g$ for $10 \mathrm{~min}$ at $4{ }^{\circ} \mathrm{C}$ and incubated on pre-equilibrated magnetic beads with anti-GFP (Chromotek) or anti-HA (Pierce) antibody for $2.5 \mathrm{~h}$ at $4{ }^{\circ} \mathrm{C}$. After incubation, the beads were washed five times with IP buffer and immunoprecipitated proteins were eluted from beads by incubation with $2 \times$ Laemmli buffer supplemented with $10 \% \beta$-mercaptoethanol for $5 \mathrm{~min}$ at $95^{\circ} \mathrm{C}$. Samples were then analyzed by western blot. For western blot analysis, protein extracts obtained from 30 embryos crushed in $30 \mu \mathrm{L}$ of $2 \times$ Laemmli buffer supplemented with $10 \% \quad \beta$ mercaptoethanol were boiled for $5 \mathrm{~min}$ at $95^{\circ} \mathrm{C}$. Samples were then loaded onto 10\% SDS-PAGE gels before transfer to a nitrocellulose membrane. The membrane was blocked for $1 \mathrm{~h}$ in $5 \%$ milk diluted in $1 \times$ PBS, $0.1 \%$ Tween 20 before proceeding to primary antibody incubation (overnight, $4{ }^{\circ} \mathrm{C}$ on a rotating plate). Antibodies and dilutions for western blot were: rabbit anti-Nos (1:1000, gift from A. Nakamura), rabbit anti-Osk (1:2000, gift from P. Lasko), mouse anti-Aub (4D10, 1:5000), ${ }^{68}$ guinea pig anti-Smg (1:2000, gift from C. Smibert), rabbit anti-PABP (1:500, gift from A. Vincent), rabbit anti-GFP (1:1000, Invitrogen), anti-HA (1:1000, Covance) and mouse anti-a-Tubulin (1:5000, Sigma). After washes in $1 \times$ PBS, $0.1 \%$ Tween 20 , the membrane was incubated for $1 \mathrm{~h}$ at room temperature with secondary antibody coupled with HRP (Jackson ImmunoResearch). After washes, HRP-conjugated secondary antibodies were revealed by chemiluminescent detection (Pierce). Quantifications were performed with the ImageJ software using the Gels tool.

\section{Mass spectrometry}

Total protein elute was loaded on 10\% SDS-PAGE gels (MiniProtean TGX Precast gels, Bio-Rad). For each sample, one band was cut after stacking migration. Gel pieces were destained with three washes in $50 \%$ acetonitrile and $50 \mathrm{mM}$ TEABC (trimethy ammonium bicarbonate buffer). After protein reduction $(10 \mathrm{mM}$ DTT in $50 \mathrm{mM}$ TEABC at $60^{\circ} \mathrm{C}$ for $30 \mathrm{~min}$ ) and alkylation $(55 \mathrm{mM}$ iodoacetamide in TEABC at room temperature in the dark for $30 \mathrm{~min}$ ), proteins were in-gel digested using $1 \mu \mathrm{g}$ Trypsin (Trypsin Gold, Promega). Digested products were dehydrated in a vacuum 
centrifuge. Obtained peptides were analyzed online using QExactive Plus mass spectrometer (Thermo Fisher Scientific) interfaced with a nano-flow HPLC (RSLC U3000, Thermo Fisher Scientific). Samples were loaded onto a $15 \mathrm{~cm}$ reverse phase column (Acclaim Pepmap 100, NanoViper, Thermo Fisher Scientific) and separated using a 103-min gradient of $2 \%-40 \%$ of buffer B ( $80 \%$ acetonitrile, $0.1 \%$ formic acid) at a flow rate of $300 \mathrm{~nL} / \mathrm{min}$. MS/MS analyses were performed in a data-dependant mode (Xcalibur software 4.1, Thermo Fisher Scientific). Full scans $(375-1500 \mathrm{~m} / \mathrm{z})$ were acquired in the Orbitrap mass analyzer with a 70,000 resolution at $200 \mathrm{~m} / \mathrm{z}$. The twelve most intense ions (charge states $\geq 2$ ) were sequentially isolated and fragmented by HCD (high-energy collisional dissociation) in the collision cell and detected at 17,500 resolution. The spectral data were analyzed using the Maxquant software (v1.5.5.1) with default settings. ${ }^{69}$ All MS/MS spectra were searched by the Andromeda search engine against a decoy database consisting of a combination of Drosophila melanogaster entries from Reference Proteome (UP000000803, release 2018_02, https://www.uniprot.org/), isoform $C$ sequence of Aub protein and classical contaminants, containing forward and reverse entries. Default search parameters were used; Oxidation (Met) and Acetylation ( $\mathrm{N}$-term) as variable modifications and Carbamidomethyl (Cys) as fixed modification were applied. FDR was set to $1 \%$ for peptides and proteins. A representative protein ID in each protein group was automatically selected using in-house bioinformatics tool (Leading_v3.2). First, proteins with the most numerous identified peptides are isolated in a "match group" (proteins from the "Protein IDs" column with the maximum number of "peptide counts"). For the match groups where more than one protein ID are present after filtering, the best annotated protein in UniProtKB, release 2019_01 (reviewed entries rather than automatic ones, highest evidence for protein existence) is defined as the "leading" protein. Label free quantification (MaxQuant LFQ) was used to identify differential proteins between samples.

\section{LUMIER assays}

S2R + cells $(250,000)$ were transfected using Effectene transfection reagent (Qiagen) and incubated for $48 \mathrm{~h}$ at $25^{\circ} \mathrm{C}$. Cells were lysed in HNTG buffer (20 mM HEPES, $150 \mathrm{mM} \mathrm{NaCl}, 1 \mathrm{mM} \mathrm{MgCl}, 1 \mathrm{mM}$ EGTA, 1\% Triton, $10 \%$ glycerol) complemented with protease inhibitor (cOmplete ${ }^{\mathrm{TM}}$ EDTA-free Protease Inhibitor Cocktail, Roche). On a plate (LUMITRAC 60096 W Microplate High Binding, Greiner) pre-coated with anti-FLAG antibody (Sigma, F1804) and blocked for $1 \mathrm{~h}$ with a blocking solution (3\% BSA, $5 \%$ sucrose and $0.5 \%$ Tween $20)$, lysates were incubated for $3 \mathrm{~h}$ on ice. After washes with HNTG, luminescence was revealed using Dual-Luciferase Reporter Assay System (Promega, E1910) and read on a luminometer Tristar LB941. Transfections were repeated 8-32 times.

Polysome profiling

For lysis of embryos, we used either of two methods that produce similar results. Either $0.2 \mathrm{~g}$ of fresh $0-2 \mathrm{~h}$ embryos were homogenized in lysis buffer composed of $20 \mathrm{mM}$ Tris- $\mathrm{HCl}, 140 \mathrm{mM} \mathrm{KCl}$, $5 \mathrm{mM} \mathrm{MgCl}_{2}, 0.5 \mathrm{mM}$ DTT, 1\% Triton X-100, $0.02 \mathrm{U} / \mu \mathrm{L}$ RNasin, 1X protease inhibitor and $0.1 \mathrm{mg} / \mathrm{mL}$ cycloheximide, or $0.2 \mathrm{~g}$ of frozen $0-2 \mathrm{~h}$ embryos were homogenized in lysis buffer composed of $30 \mathrm{mM}$ Tris- $\mathrm{HCl}, 100 \mathrm{mM} \mathrm{NaCl}, 10 \mathrm{mM} \mathrm{MgCl} 2,0.5 \mathrm{mM}$ DTT, $1 \%$ Triton $\mathrm{X}-100,0.02 \mathrm{U} / \mu \mathrm{L}$ RNasin, $1 \times$ protease inhibitor and $0.1 \mathrm{mg} / \mathrm{mL}$ cycloheximide. Embryo extracts were incubated for $30 \mathrm{~min}$ on ice. Homogenates were cleared by full speed centrifugation for $30 \mathrm{~min}$ at $4{ }^{\circ} \mathrm{C}$. For treatment with puromycin, fresh embryos were homogenized in lysis buffer composed of $20 \mathrm{mM}$ Tris- $\mathrm{HCl}, 140 \mathrm{mM} \mathrm{KCl}$, $0.5 \mathrm{mM}$ DTT, 1\% Triton X-100, $0.02 \mathrm{U} / \mu \mathrm{L}$ RNasin, $1 \times$ protease inhibitor and $2 \mathrm{mM}$ puromycin. Extracts were incubated for $20 \mathrm{~min}$ on ice followed by $20 \mathrm{~min}$ at $37^{\circ} \mathrm{C}$ and cleared as before. The amount of RNA in the extracts was quantified using Nanodrop. Volumes of extract containing equal amounts of RNA were loaded on top of
$10 \%-50 \%$ sucrose gradient containing cycloheximide except for puromycin-treated samples. Gradients were centrifuged for $2 \mathrm{~h}$ at $34,000 \mathrm{rpm}$ in a SW41 rotor, with no brake. $1 \mathrm{~mL}$ fractions were collected using a ISCO gradient collector. $900 \mu \mathrm{L}$ of each fraction were used for RNA extraction and $100 \mu \mathrm{L}$ for protein precipitation. For RNA extraction, $500 \mathrm{pg}$ of luciferase RNA was added to $900 \mu \mathrm{L}$ of fraction to control RNA extraction. $0.5 \%$ SDS and $10 \mathrm{mM}$ EDTA was then added to each fraction, followed by a 5 min incubation. Then, RNAs were prepared using acid phenol-chloroform. One volume of acid phenol-chloroform was added, and the mixture was vortexed and centrifuged at $12,000 \times g$ for $15 \mathrm{~min}$. The aqueous phase was put in a new tube with $2 \mu \mathrm{L}$ of glycoblue and 1 volume of isopropanol, incubated for $15 \mathrm{~min}$ and centrifuged for $15 \mathrm{~min}$ at $12,000 \times \mathrm{g}$. The RNA pellet was washed twice with 1 volume of $75 \%$ ethanol. The RNA pellet was dried for $5 \mathrm{~min}$ and resuspended in $20 \mu \mathrm{L}$ of RNasefree $\mathrm{H}_{2} \mathrm{O} .5 \mu \mathrm{L}$ of each RNA sample were used for CDNA synthesis using superscript III and random primers. CDNAs were diluted at 1:10 for qPCR reactions that were performed using LightCycler 480 (Roche) and the primers are listed in Supplementary information, Table S3. Data were analyzed using the $\Delta \mathrm{Cp}$ method. ${ }^{70}$ For protein preparation, $400 \mu \mathrm{L}$ of methanol and $100 \mu \mathrm{L}$ of chloroform were added to $100 \mu \mathrm{L}$ of fraction and the mixture was vortexed. The mixture was then added with $300 \mu \mathrm{L}$ of $\mathrm{H}_{2} \mathrm{O}$, vortexed and centrifuged at full speed for $5 \mathrm{~min}$. The upper phase was discarded, $435 \mu \mathrm{L}$ of methanol were added and the mixture was centrifuged at full speed for $5 \mathrm{~min}$. The protein pellet was dried for $1-2 \mathrm{~min}$ and resuspended in $100 \mu \mathrm{L}$ of $2 \times$ Laemmli buffer supplemented with $10 \% \beta$-mercaptoethanol. Proteins were analyzed by western blot; antibodies and dilutions were mouse anti-Aub (4D10, 1:5000), ${ }^{68}$ rabbit anti-PABP (1:1000, gift from A. Vincent), rabbit anti-RpL10Ab $(1: 5000)^{71}$ and rabbit anti-RpS3 (1:1000). ${ }^{71}$

\section{Cloning and recombineering}

To produce the UASp-HA-elF3d and UASp-HA-elF3d $d^{\text {helix } 11}$ transgenes, elF3d and elF3 $\mathrm{d}^{\text {helix } 11}$ coding sequences were amplified by PCR from clones provided by S. Rumpf. ${ }^{49}$ PCR fragments were cloned into pENTR by directional Topo cloning (Invitrogen). The generated plasmids were used in gateway cloning to insert the sequences into pPHW (UASp-HA-attR1-ccdB-attR2-SV40 $3^{\prime} U T R$ ) in which an attB (pPHW-attB) site has been inserted. The resulting fragments were then inserted in the Drosophila genome by PhiC31 recombination into the attP40 site (BestGene).

To produce FLAG-FFL-Aub, Aub-coding sequence was PCR amplified from the p8161 plasmid ${ }^{66}$ and cloned into pENTR by directional Topo cloning (Invitrogen). The resulting plasmid was used in gateway cloning to insert Aub sequence into pAct-FLAGFirefly-RfA ${ }^{72}$ (pAFW (DGRC) in which the FFL-coding sequence has been added). To produce HA-RL tagged versions of elF3b (DGRC, FI08008), elF3d, ${ }^{49}$ elF3f (DGRC, LD47792), elF3k (DGRC, LD03569) and eIF4E (from E. Wahle), the coding sequences were amplified by PCR and cloned into pENTR by directional Topo cloning. The resulting plasmids were used in gateway cloning to insert the coding sequences into pAct-HA-Renilla-RfA ${ }^{72}$ (pAHW (DGRC) in which the RL-coding sequence has been added). For the HA-RL tagged versions of PABP (DNASU, DmCD00772781), elF3g (DNASU, DmCD00766429), elF3h (DNASU, DmCD00764259) and elF4a (DNASU, DmCD00764657), plasmids were directly used in gateway cloning to insert the sequence into pAct-HA-Renilla-RfA. FLAG-FFL-Cherry, HA-RL-Cherry and Sd-RL-HA ${ }^{72}$ were used as negative controls. To produce GST-PABP clones, the coding sequences of five $p A b p$ domains, RRM1, RRM2, RRM3, RRM4 and PABC were amplified by PCR from a plasmid provided by E. Wahle. A stop codon (TAA) was added at the end of each domain. The different fragments were cloned into pGEX-4T-1 (Sigma) digested with EcoRl and Xhol. The plasmids containing HA-Aub(1-482) and HA-Aub(476-866) fragments were generated previously. ${ }^{13}$ The primers used to generate the constructs and the constructs are listed in Supplementary information, Tables S3 and 4, respectively. 
GST pull-down assays

The plasmids containing GST-RRM1, GST-RRM2, GST-RRM3, GST-RRM4 and GST-PABC were introduced in E. coli BL21. Protein production was induced by IPTG treatment overnight at $18^{\circ} \mathrm{C}$, or at $37^{\circ} \mathrm{C}$ for GSTRRM2. GST-fused proteins were affinity-purified on glutathioneSepharose $4 \mathrm{~B}$ beads (GE Healthcare); the beads were incubated overnight at $4{ }^{\circ} \mathrm{C}$ in PBT, cOmplete ${ }^{\mathrm{TM}}$ EDTA-free Protease Inhibitor Cocktail (Roche) and 5\% BSA. HA-Aub proteins were synthesized in vitro using the TnT Coupled reticulocyte lysate system (Promega), and were incubated with immobilized GST fusion proteins in $400 \mu \mathrm{L}$ binding buffer ( $50 \mathrm{mM}$ HEPES, pH 7.5, $500 \mathrm{mM} \mathrm{NaCl}, 0.2 \mathrm{mM}$ EDTA, $1 \mathrm{mM}$ DTT, 0.5\% NP-40, cOmplete ${ }^{\mathrm{TM}}$ EDTA-free Protease Inhibitor Cocktail (Roche)) containing $0.2 \mu \mathrm{g} / \mu \mathrm{L}$ RNase A. Incubations were performed for $1 \mathrm{~h}$ at $4{ }^{\circ} \mathrm{C}$, followed by $30 \mathrm{~min}$ at room temperature. Glutathione-Sepharose beads were then washed four times with binding buffer at room temperature. Recombinant proteins were dissociated from the beads by boiling for $5 \mathrm{~min}$ in Laemmli buffer and separated on a SDS-PAGE gel. Western blots were revealed with mouse anti-HA antibody (Covance, MMS-101R) at dilution of 1:1000.

Quantification and statistical analysis

Statistical analysis of mass spectrometric data. Individual LFQ values per detected peptides were first quantile normalized given the experimental condition by using the ProStar (prostar-proteomics. org) ${ }^{73}$ software with the default parameter set. After normalization an imputation step was applied in cases where only one value was missing in each condition group by replacing the missing data by the mean of the observed value for this peptide in their respective experimental condition. Then, each individual experiment was combined into one data matrix. To account for batch effects, ComBat from the R package sva was used. After quality controls, differential expression analysis was done using Reproducibility-Optimized Test Statistic (ROTS) ${ }^{74}$ for each different comparison. P-values and FDR were extracted and plotted using self-written R scripts. Significant proteins were annotated using the FlyMine database. ${ }^{75}$

Immunofluorescence quantification. Fluorescent images were acquired using a Leica SP8 confocal scanning microscope. Quantification of fluorescent signal was performed using ImageJ tool Measure.

Colocalization quantification. Quantification of colocalization in Fig. 2 was performed in 3D using the Imaris software. For colocalization in granules (around nuclei), spots were defined with a minimal size of $0.5 \mu \mathrm{m}$ and a PSF correction was applied to account for confocal acquisition deformation. Spot colocalization was determined within a radius of $0.25 \mu \mathrm{m}$ around the center of the spot. For colocalization in foci (between nuclei), spots were defined with a minimal size of $0.2 \mu \mathrm{m}$ and a PSF correction was applied to account for confocal acquisition deformation. Spot colocalization was determined within a radius of $0.25 \mu \mathrm{m}$ around the center of the spot. Quantification of colocalization and overlapping signals in Supplementary information, Fig. S3 was performed using ImageJ, with four embryos per staining. Lines were drawn across each GFP-Aub germ granules to obtain the intensity profiles of GFP-Aub and PABP, or GFP-Aub and HA-elF3d; background signal was subtracted. Each GFP-Aub peak was manually categorized as colocalized, overlapping (single or double) or separated with peaks from the other channel, as depicted in Supplementary information, Fig. S3a, d.

\section{DATA AVAILABILITY}

The mass spectrometry proteomics data have been deposited to the ProteomeXchange Consortium via the PRIDE $^{76}$ partner repository with the dataset identifier PXD016399.

\section{ACKNOWLEDGEMENTS}

We thank S. Dorner, A. Nakamura, P. Lasko, M. Siomi, C. Smibert and A. Vincent for their gifts of antibodies, and A. Ephrussi, E. Gavis, F. Gebauer, S. Rumpf and E. Wahle for their gifts of fly stocks and DNA clones. This work was supported by the CNRSUniversity of Montpellier UMR9002, ANR (ANR-15-CE12-0019-01), FRM ("Equipe FRM 2013 DEQ20130326534"). A.R. was supported by the Labex EpiGenMed/University of Montpellier (ANR-10-LABX-12-01) and the Fondation ARC, and C.J. by the Labex EpiGenMed. M.-R.G.-S., J.D., C.G., J.C. and V.P. held a salary from ANR, and R.N.-S. from AFM-Telethon and FRM.

\section{AUTHOR CONTRIBUTIONS}

M.S. designed the study. A.R., M.-R.G.-S., C.J., R.N.-S., J.D., C.G. and A.C. conducted the experiments and analyzed the data; V.P. and J.C. performed bioinformatic analyses; M.D. performed mass spectrometry experiments; A.B. helped with polysome gradients; F.J. produced clones and provided advices for LUMIER assays. M.S. and A.R. wrote the manuscript; all authors commented on the manuscript.

\section{ADDITIONAL INFORMATION}

Supplementary information accompanies this paper at https://doi.org/10.1038/ s41422-020-0294-9.

Competing interests: The authors declare no competing interests.

\section{REFERENCES}

1. Barckmann, B. \& Simonelig, M. Control of maternal mRNA stability in germ cells and early embryos. Biochim. Biophys. Acta 1829, 714-724 (2013).

2. Gavis, E. R. \& Lehmann, R. Localization of nanos RNA controls embryonic polarity. Cell 71, 301-313 (1992).

3. Bergsten, S. E. \& Gavis, E. R. Role for mRNA localization in translational activation but not spatial restriction of nanos RNA. Development 126, 659-669 (1999).

4. Trcek, T. et al. Drosophila germ granules are structured and contain homotypic mRNA clusters. Nat. Commun. 6, 7962 (2015).

5. Dahanukar, A. \& Wharton, R. P. The Nanos gradient in Drosophila embryos is generated by translational regulation. Genes Dev. 10, 2610-2620 (1996).

6. Gavis, E. R. \& Lehmann, R. Translational regulation of nanos by RNA localization. Nature 369, 315-318 (1994).

7. Dahanukar, A., Walker, J. A. \& Wharton, R. P. Smaug, a novel RNA-binding protein that operates a translational switch in Drosophila. Mol. Cell 4, 209-218 (1999).

8. Smibert, C. A., Wilson, J. E., Kerr, K. \& Macdonald, P. M. Smaug protein represses translation of unlocalized nanos mRNA in the Drosophila embryo. Genes Dev. 10, 2600-2609 (1996).

9. Zaessinger, S., Busseau, I. \& Simonelig, M. Oskar allows nanos mRNA translation in Drosophila embryos by preventing its deadenylation by Smaug/CCR4. Development 133, 4573-4583 (2006).

10. Ephrussi, A. \& Lehmann, R. Induction of germ cell formation by oskar. Nature $\mathbf{3 5 8}$, 387-392 (1992)

11. Jeske, M., Moritz, B., Anders, A. \& Wahle, E. Smaug assembles an ATP-dependent stable complex repressing nanos mRNA translation at multiple levels. EMBO J. 30, 90-103 (2011).

12. Barckmann, B. et al. Aubergine iCLIP reveals piRNA-dependent decay of mRNAs involved in germ cell development in the early embryo. Cell Rep. 12, 1205-1216 (2015).

13. Dufourt, J. et al. piRNAs and Aubergine cooperate with Wispy poly(A) polymerase to stabilize mRNAs in the germ plasm. Nat. Commun. 8, 1305 (2017).

14. Czech, B. et al. piRNA-guided genome defense: from biogenesis to silencing. Annu. Rev. Genet. 52, 131-157 (2018).

15. Huang, X., Fejes Toth, K. \& Aravin, A. A. piRNA biogenesis in Drosophila melanogaster. Trends Genet. 33, 882-894 (2017).

16. Rojas-Rios, P. \& Simonelig, M. piRNAs and PIWI proteins: regulators of gene expression in development and stem cells. Development 145, pii: dev161786 (2018).

17. Mani, S. R., Megosh, H. \& Lin, H. PIWI proteins are essential for early Drosophila embryogenesis. Dev. Biol. 385, 340-349 (2014).

18. Rouget, $C$. et al. Maternal mRNA deadenylation and decay by the piRNA pathway in the early Drosophila embryo. Nature 467, 1128-1132 (2010).

19. Vourekas, A., Alexiou, P., Vrettos, N., Maragkakis, M. \& Mourelatos, Z. Sequencedependent but not sequence-specific piRNA adhesion traps mRNAs to the germ plasm. Nature 531, 390-394 (2016).

20. Goh, W. S. et al. piRNA-directed cleavage of meiotic transcripts regulates spermatogenesis. Genes Dev. 29, 1032-1044 (2015).

21. Gou, L. T. et al. Pachytene piRNAs instruct massive mRNA elimination during late spermiogenesis. Cell Res. 24, 680-700 (2014). 
22. Kiuchi, T. et al. A single female-specific piRNA is the primary determiner of sex in the silkworm. Nature 509, 633-636 (2014).

23. Watanabe, T., Cheng, E. C., Zhong, M. \& Lin, H. Retrotransposons and pseudogenes regulate mRNAs and IncRNAs via the piRNA pathway in the germline. Genome Res. 25, 368-380 (2015).

24. Zhang, P. et al. MIWI and piRNA-mediated cleavage of messenger RNAs in mouse testes. Cell Res. 25, 193-207 (2015).

25. Riechmann, V., Gutierrez, G. J., Filardo, P., Nebreda, A. R. \& Ephrussi, A. Par-1 regulates stability of the posterior determinant Oskar by phosphorylation. Nat. Cell Biol. 4, 337-342 (2002).

26. Markussen, F. H., Michon, A. M., Breitwieser, W. \& Ephrussi, A. Translational control of oskar generates short OSK, the isoform that induces pole plasma assembly. Development 121, 3723-3732 (1995).

27. Malone, C. D. et al. Specialized piRNA pathways act in germline and somatic tissues of the Drosophila ovary. Cell 137, 522-535 (2009).

28. Sienski, G. et al. Silencio/CG9754 connects the Piwi-piRNA complex to the cellular heterochromatin machinery. Genes Dev. 29, 2258-2271 (2015).

29. $\mathrm{Yu}, \mathrm{Y}$. et al. Panoramix enforces piRNA-dependent cotranscriptional silencing. Science 350, 339-342 (2015).

30. Khurana, J. S., Xu, J., Weng, Z. \& Theurkauf, W. E. Distinct functions for the Drosophila piRNA pathway in genome maintenance and telomere protection. PLoS Genet. 6, e1001246 (2010).

31. Little, S. C., Sinsimer, K. S., Lee, J. J., Wieschaus, E. F. \& Gavis, E. R. Independent and coordinate trafficking of single Drosophila germ plasm mRNAs. Nat. Cell Biol. 17, 558-568 (2015).

32. Thomson, T., Liu, N., Arkov, A., Lehmann, R. \& Lasko, P. Isolation of new polar granule components in Drosophila reveals $\mathrm{P}$ body and ER associated proteins. Mech. Dev. 125, 865-873 (2008).

33. Kirino, Y. et al. Arginine methylation of Aubergine mediates Tudor binding and germ plasm localization. RNA 16, 70-78 (2010).

34. Nishida, K. M. et al. Functional involvement of Tudor and dPRMT5 in the piRNA processing pathway in Drosophila germlines. EMBO J. 28, 3820-3831 (2009).

35. Gotze, $M$. et al. Translational repression of the Drosophila nanos mRNA involves the RNA helicase Belle and RNA coating by Me31B and Trailer hitch. RNA 23, 1552-1568 (2017).

36. Kirino, Y. et al. Arginine methylation of Piwi proteins catalysed by dPRMT5 is required for Ago3 and Aub stability. Nat. Cell Biol. 11, 652-658 (2009).

37. Trepte, P. et al. DULIP: a dual luminescence-based co-immunoprecipitation assay for interactome mapping in mammalian cells. J. Mol. Biol. 427, 3375-3388 (2015).

38. Brook, M., Smith, J. W. \& Gray, N. K. The DAZL and PABP families: RNA-binding proteins with interrelated roles in translational control in oocytes. Reproduction 137, 595-617 (2009).

39. Valasek, L. S. et al. Embraced by elF3: structural and functional insights into the roles of elF3 across the translation cycle. Nucleic Acids Res. 45, 10948-10968 (2017).

40. Lee, A. S., Kranzusch, P. J. \& Cate, J. H. elF3 targets cell-proliferation messenger RNAs for translational activation or repression. Nature 522, 111-114 (2015).

41. Lee, A. S., Kranzusch, P. J., Doudna, J. A. \& Cate, J. H. elF3d is an mRNA capbinding protein that is required for specialized translation initiation. Nature $\mathbf{5 3 6}$ 96-99 (2016).

42. Beilharz, T. H. \& Preiss, T. Translational profiling: the genome-wide measure of the nascent proteome. Brief Funct. Genom. Proteom. 3, 103-111 (2004).

43. Benoit, B. et al. An essential role for the RNA-binding protein Smaug during the Drosophila maternal-to-zygotic transition. Development 136, 923-932 (2009).

44. Tadros, W. et al. SMAUG is a major regulator of maternal mRNA destabilization in Drosophila and its translation is activated by the PAN GU kinase. Dev. Cell 12, 143-155 (2007).

45. Kronja, I. et al. Widespread changes in the posttranscriptional landscape at the Drosophila oocyte-to-embryo transition. Cell Rep. 7, 1495-1508 (2014).

46. Grivna, S. T., Pyhtila, B. \& Lin, H. MIWI associates with translational machinery and PIWl-interacting RNAs (piRNAs) in regulating spermatogenesis. Proc. Natl. Acad. Sci. USA 103, 13415-13420 (2006)

47. Unhavaithaya, Y. et al. MILI, a PIWI-interacting RNA-binding protein, is required for germ line stem cell self-renewal and appears to positively regulate translation. J. Biol. Chem. 284, 6507-6519 (2009).

48. Szostak, E. et al. Hrp48 and elF3d contribute to msl-2 mRNA translational repression. Nucleic Acids Res. 46, 4099-4113 (2018).

49. Rode, S. et al. Differential requirement for translation initiation factor pathways during ecdysone-dependent neuronal remodeling in Drosophila. Cell Rep. 24 2287-2299 (2018).

50. Meyer, K. D. et al. 5' UTR m(6)A promotes cap-independent translation. Cell 163, 999-1010 (2015)

51. Thakor, N. et al. Cellular mRNA recruits the ribosome via elF3-PABP bridge to initiate internal translation. RNA Biol. 14, 553-567 (2017).
52. Nelson, M. R., Leidal, A. M. \& Smibert, C. A. Drosophila cup is an elF4E-binding protein that functions in Smaug-mediated translational repression. EMBO J. 23 150-159 (2004).

53. Igreja, C. \& Izaurralde, E. CUP promotes deadenylation and inhibits decapping of mRNA targets. Genes Dev. 25, 1955-1967 (2011).

54. Chen, Y. et al. A DDX6-CNOT1 complex and W-binding pockets in CNOT9 reveal direct links between miRNA target recognition and silencing. Mol. Cell 54, 737-750 (2014).

55. Mathys, $\mathrm{H}$. et al. Structural and biochemical insights to the role of the CCR4-NOT complex and DDX6 ATPase in microRNA repression. Mol. Cell 54, 751-765 (2014).

56. Dodson, A. E. \& Kennedy, S. Germ granules coordinate RNA-based epigenetic inheritance pathways. Dev. Cell 50, 704-715 (2019).

57. Ouyang, J. P. T. et al. P granules protect RNA interference genes from silencing by piRNAs. Dev. Cell 50, 716-728 (2019).

58. Rangan, P. et al. Temporal and spatial control of germ-plasm RNAs. Curr. Biol. 19, 72-77 (2009)

59. Weil, T. T. et al. Drosophila patterning is established by differential association of mRNAs with P bodies. Nat. Cell Biol. 14, 1305-1313 (2012).

60. Dai, P. et al. A translation-activating function of MIWI/piRNA during mouse spermiogenesis. Cell 179, 1566-1581 (2019).

61. Schupbach, T. \& Wieschaus, E. Female sterile mutations on the second chromosome of Drosophila melanogaster. II. Mutations blocking oogenesis or altering egg morphology. Genetics 129, 1119-1136 (1991).

62. Rorth, P. Gal4 in the Drosophila female germline. Mech. Dev. 78, 113-118 (1998).

63. Tomari, Y. et al. RISC assembly defects in the Drosophila RNAi mutant armitage. Cell 116, 831-841 (2004).

64. Cook, H. A., Koppetsch, B. S., Wu, J. \& Theurkauf, W. E. The Drosophila SDE3 homolog armitage is required for oskar mRNA silencing and embryonic axis specification. Cell 116, 817-829 (2004).

65. Forrest, K. M., Clark, I. E., Jain, R. A. \& Gavis, E. R. Temporal complexity within a translational control element in the nanos mRNA. Development 131, 5849-5857 (2004).

66. Harris, A. N. \& Macdonald, P. M. Aubergine encodes a Drosophila polar granule component required for pole cell formation and related to elF2C. Development 128, 2823-2832 (2001)

67. Chartier, A. et al. Mitochondrial dysfunction reveals the role of mRNA poly(A) tail regulation in oculopharyngeal muscular dystrophy pathogenesis. PLoS Genet. 11 e1005092 (2015).

68. Gunawardane, L. S. et al. A slicer-mediated mechanism for repeat-associated siRNA $5^{\prime}$ end formation in Drosophila. Science 315, 1587-1590 (2007).

69. Cox, J. \& Mann, M. MaxQuant enables high peptide identification rates, individualized p.p.b.-range mass accuracies and proteome-wide protein quantification. Nat. Biotechnol. 26, 1367-1372 (2008).

70. Panda, A. C., Martindale, J. L. \& Gorospe, M. Polysome fractionation to analyze mRNA distribution profiles. Bio-Protocol 7, pii: e2126 (2017).

71. Antic, S., Wolfinger, M. T., Skucha, A., Hosiner, S. \& Dorner, S. General and microRNA-mediated mRNA degradation occurs on ribosome complexes in Drosophila cells. Mol. Cell Biol. 35, 2309-2320 (2015).

72. Srivastava, D., de Toledo, M., Manchon, L., Tazi, J. \& Juge, F. Modulation of Yorkie activity by alternative splicing is required for developmental stability. https://doi. org/10.1101/2019.12.19.882779 (2019).

73. Wieczorek, S. et al. DAPAR \& ProStaR: software to perform statistical analyses in quantitative discovery proteomics. Bioinformatics 33, 135-136 (2017).

74. Suomi, T., Seyednasrollah, F., Jaakkola, M. K., Faux, T. \& Elo, L. L. ROTS: an R package for reproducibility-optimized statistical testing. PLoS Comput. Biol. 13, e1005562 (2017).

75. Lyne, R. et al. FlyMine: an integrated database for Drosophila and Anopheles genomics. Genome Biol. 8, R129 (2007).

76. Perez-Riverol, Y. et al. The PRIDE database and related tools and resources in 2019: improving support for quantification data. Nucleic Acids Res. 47, D442-D450 (2019).

(i) Open Access This article is licensed under a Creative Commons Attribution 4.0 International License, which permits use, sharing, adaptation, distribution and reproduction in any medium or format, as long as you give appropriate credit to the original author(s) and the source, provide a link to the Creative Commons license, and indicate if changes were made. The images or other third party material in this article are included in the article's Creative Commons license, unless indicated otherwise in a credit line to the material. If material is not included in the article's Creative Commons license and your intended use is not permitted by statutory regulation or exceeds the permitted use, you will need to obtain permission directly from the copyright holder. To view a copy of this license, visit http://creativecommons. org/licenses/by/4.0/.

(c) The Author(s) 2020 